\title{
Non-linear complementary filters on the special orthogonal group
}

\author{
Robert Mahony, Member, IEEE, Tarek Hamel, Member, IEEE, and Jean-Michel Pflimlin, Member, IEEE
}

\begin{abstract}
This paper considers the problem of obtaining good attitude estimates from measurements obtained from typical low cost inertial measurement units. The outputs of such systems are characterised by high noise levels and time varying additive biases. We formulate the filtering problem as deterministic observer kinematics posed directly on the special orthogonal group $S O(3)$ driven by reconstructed attitude and angular velocity measurements. Lyapunov analysis results for the proposed observers are derived that ensure almost global stability of the observer error. The approach taken leads to an observer that we term the direct complementary filter. By exploiting the geometry of the special orthogonal group a related observer, termed the passive complementary filter, is derived that decouples the gyro measurements from the reconstructed attitude in the observer inputs. Both the direct and passive filters can be extended to estimate gyro bias on-line. The passive filter is further developed to provide a formulation in terms of the measurement error that avoids any algebraic reconstruction of the attitude. This leads to an observer on $S O(3)$, termed the explicit complementary filter, that requires only accelerometer and gyro outputs; is suitable for implementation on embedded hardware; and provides good attitude estimates as well as estimating the gyro biases on-line. The performance of the observers are demonstrated with a set of experiments performed on a robotic test-bed and a radio controlled unmanned aerial vehicle.
\end{abstract}

Index Terms-Complementary filter, nonlinear observer, attitude estimates, special orthogonal group.

\section{INTRODUCTION}

$\mathbf{T}$ HE recent proliferation of Micro-Electro-Mechanical Systems (MEMS) components has lead to the development of a range of low cost and light weight inertial measurement units. The low power, light weight and potential for low cost manufacture of these units opens up a wide range of applications in areas such as virtual reality and gaming systems, robotic toys, and low cost mini-aerialvehicles (MAVs) such as the Hovereye (Fig. 1). The signal output of low cost IMU systems, however, is characterised by low-resolution signals subject to high noise levels as well as general time-varying bias terms. The raw signals must be processed to reconstruct smoothed attitude estimates and biascorrected angular velocity measurements. For many of the low cost applications considered the algorithms need to run on embedded processors with low memory and processing resources.

R. Mahony is with Department of Engineering, Australian National University, ACT, 0200, Australia. e-mail: Robert.Mahony@anu.edu.au.

T. Hamel is with I3S-CNRS, Nice-Sophia Antipolis, France. e-mail: thamel@i3s.unice.fr.

J.-M. Pflimlin is with Department of Navigation, Dassault Aviation, Saint Cloud, Paris. France. e-mail: Jean-Michel.Pflimlin@dassault-aviation.com.

Manuscript received November 08, 2006; revised August 03, 2007.
There is a considerable body of work on attitude reconstruction for robotics and control applications (for example [1]-[4]). A standard approach is to use extended stochastic linear estimation techniques [5], [6]. An alternative is to use deterministic complementary filter and non-linear observer design techniques [7]-[9]. Recent work has focused on some of the issues encountered for low cost IMU systems [9][12] as well as observer design for partial attitude estimation [13]-[15]. It is also worth mentioning the related problem of fusing IMU and vision data that is receiving recent attention [16]-[19] and the problem of fusing IMU and GPS data [9], [20]. Parallel to the work in robotics and control there is a significant literature on attitude heading reference systems (AHRS) for aerospace applications [21]. An excellent review of attitude filters is given by Crassidis et al. [22]. The recent interest in small low-cost aerial robotic vehicles has lead to a renewed interest in lightweight embedded IMU systems [8], [23]-[25]. For the low-cost light-weight systems considered, linear filtering techniques have proved extremely difficult to apply robustly [26] and linear single-input single-output complementary filters are often used in practice [25], [27]. A key issue is on-line identification of gyro bias terms. This problem is also important in IMU callibration for satellite systems [5], [21], [28]-[31]. An important development that came from early work on estimation and control of satellites was the use of the quaternion representation for the attitude kinematics [30], [32]-[34]. The non-linear observer designs that are based on this work have strong robustness properties and deal well with the bias estimation problem [9], [30]. However, apart from the earlier work of the authors [14], [35], [36] and some recent work on invariant observers [37], [38] there appears to be almost no work that considers the formulation of non-linear attitude observers directly on the matrix Lie-group representation of $S O(3)$.

In this paper we study the design of non-linear attitude observers on $S O(3)$ in a general setting. We term the proposed observers complementary filters because of the similarity of the architecture to that of linear complementary filters (cf. Appendix A), although, for the non-linear case we do not have a frequency domain interpretation. A general formulation of the error criterion and observer structure is proposed based on the Lie-group structure of $S O(3)$. This formulation leads us to propose two non-linear observers on $S O(3)$, termed the direct complementary filter and passive complementary filter. The direct complementary filter is closely related to recent work on invariant observers [37], [38] and corresponds (up to some minor technical differences) to non-linear observers proposed using the quaternion representation [9], [30], [32]. 


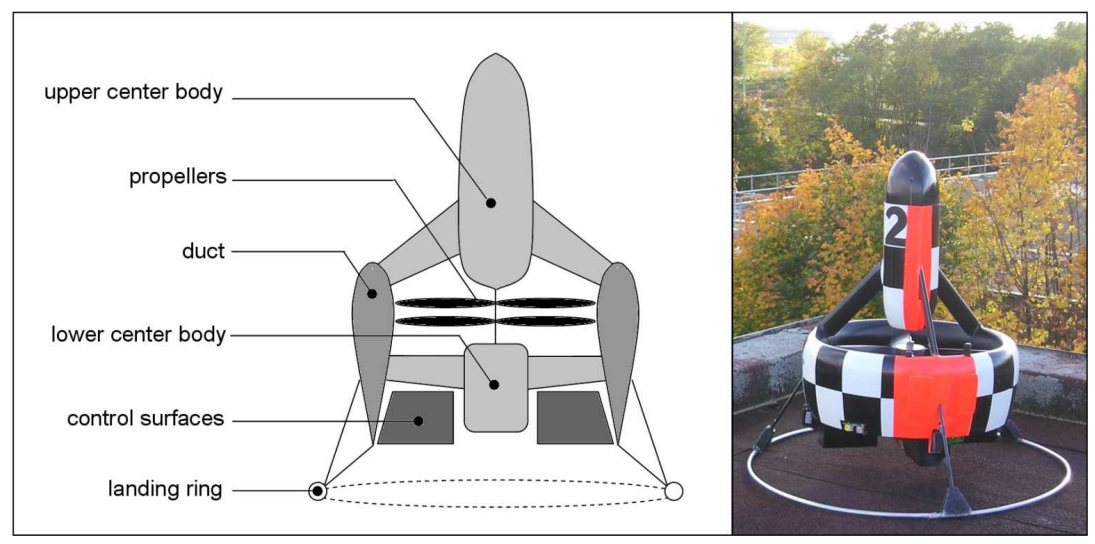

Fig. 1. The VTOL MAV HoverEye ${ }^{\complement}$ of Bertin Technologies.

We do not know of a prior reference for the passive complementary filter. The passive complementary filter has several practical advantages associated with implementation and lowsensitivity to noise. In particular, we show that the filter can be reformulated in terms of vectorial direction measurements such as those obtained directly from an IMU system; a formulation that we term the explicit complementary filter. The explicit complementary filter does not require on-line algebraic reconstruction of attitude, an implicit weakness in prior work on non-linear attitude observers [22] due to the computational overhead of the calculation and poor error characterisation of the constructed attitude. As a result the observer is ideally suited for implementation on embedded hardware platforms. Furthermore, the relative contribution of different data can be preferentially weighted in the observer response, a property that allows the designer to adjust for application specific noise characteristics. Finally, the explicit complementary filter remains well defined even if the data provided is insufficient to algebraically reconstruct the attitude. This is the case, for example, for an IMU with only accelerometer and rate gyro sensors. A comprehensive stability analysis is provided for all three observers that proves local exponential and almost global stability of the observer error dynamics, that is, a stable linearisation for zero error along with global convergence of the observer error for all initial conditions and system trajectories other than on a set of measure zero. Although the principal results of the paper are presented in the matrix Lie group representation of $S O(3)$, the equivalent quaternion representation of the observers are presented in an appendix. The authors recommend that the quaternion representations are used for hardware implementation.

The body of paper consists of five sections followed by a conclusion and two appendices. Section II provides a quick overview of the sensor model, geometry of $S O(3)$ and introduces the notation used. Section III details the derivation of the direct and passive complementary filters. The development here is deliberately kept simple to be clear. Section IV integrates on-line bias estimation into the observer design and provides a detailed stability analysis. Section V develops the explicit complementary filter, a reformulation of the passive complementary filter directly in terms of error measurements.
A suite of experimental results, obtained during flight tests of the Hovereye (Fig. 1), are provided in Section VI that demonstrate the performance of the proposed observers. In addition to the conclusion ( $\S \mathrm{VII})$ there is a short appendix on linear complementary filter design and a second appendix that provides the equivalent quaternion formulation of the proposed observers.

\section{Problem Formulation And Notation.}

\section{A. Notation and mathematical identities}

The special orthogonal group is denoted $S O(3)$. The associated Lie-algebra is the set of anti-symmetric matrices

$$
\mathfrak{s o}(3)=\left\{A \in \mathbb{R}^{3 \times 3} \mid A=-A^{T}\right\}
$$

For any two matrices $A, B \in \mathbb{R}^{n \times n}$ then the Lie-bracket (or matrix commutator) is $[A, B]=A B-B A$. Let $\Omega \in \mathbb{R}^{3}$ then we define

$$
\Omega_{\times}=\left(\begin{array}{ccc}
0 & -\Omega_{3} & \Omega_{2} \\
\Omega_{3} & 0 & -\Omega_{1} \\
-\Omega_{2} & \Omega_{1} & 0
\end{array}\right) .
$$

For any $v \in \mathbb{R}^{3}$ then $\Omega_{\times} v=\Omega \times v$ is the vector cross product. The operator vex $: \mathfrak{s o}(3) \rightarrow \mathbb{R}^{3}$ denotes the inverse of the $\Omega_{\times}$ operator

$$
\begin{aligned}
\operatorname{vex}\left(\Omega_{\times}\right) & =\Omega, & \Omega \in \mathbb{R}^{3} . \\
\operatorname{vex}(A)_{\times} & =A, & A \in \mathfrak{s o}(3)
\end{aligned}
$$

For any two matrices $A, B \in \mathbb{R}^{n \times n}$ the Euclidean matrix inner product and Frobenius norm are defined

$$
\begin{aligned}
\langle\langle A, B\rangle\rangle & =\operatorname{tr}\left(A^{T} B\right)=\sum_{i, j=1}^{n} A_{i j} B_{i j} \\
\|A\| & =\sqrt{\langle\langle A, A\rangle\rangle}=\sqrt{\sum_{i, j=1}^{n} A_{i j}^{2}}
\end{aligned}
$$


The following identities are used in the paper

$$
\begin{aligned}
(R v)_{\times} & =R v_{\times} R^{T}, & R \in S O(3), v & \in \mathbb{R}^{3} \\
(v \times w)_{\times} & =\left[v_{\times}, w_{\times}\right] & v, w & \in \mathbb{R}^{3} \\
v^{T} w=\langle v, w\rangle & =\frac{1}{2}\left\langle\left\langle v_{\times}, w_{\times}\right\rangle\right\rangle, & v, w & \in \mathbb{R}^{3} \\
v^{T} v=|v|^{2} & =\frac{1}{2}\left\|v_{\times}\right\|^{2}, & & v \in \mathbb{R}^{3} \\
\left\langle\left\langle A, v_{\times}\right\rangle\right\rangle & =0, & A=A^{T} \in \mathbb{R}^{3}, v & \in \mathbb{R}^{3} \\
\operatorname{tr}([A, B]) & =0, & A, B & \in \mathbb{R}^{3 \times 3}
\end{aligned}
$$

The following notation for frames of reference is used

- $\{A\}$ denotes an inertial (fixed) frame of reference.

- $\{B\}$ denotes a body-fixed-frame of reference.

- $\{E\}$ denotes the estimator frame of reference.

Let $\mathbb{P}_{a}, \mathbb{P}_{s}$ denote, respectively, the anti-symmetric and symmetric projection operators in square matrix space

$$
\mathbb{P}_{a}(H)=\frac{1}{2}\left(H-H^{T}\right), \quad \mathbb{P}_{s}(H)=\frac{1}{2}\left(H+H^{T}\right) .
$$

Let $(\theta, a)(|a|=1)$ denote the angle-axis coordinates of $R \in S O(3)$. One has [39]:

$$
\begin{gathered}
R=\exp \left(\theta a_{\times}\right), \quad \log (R)=\theta a_{\times} \\
\cos (\theta)=\frac{1}{2}(\operatorname{tr}(R)-1), \quad \mathbb{P}_{a}(R)=\sin (\theta) a_{\times} .
\end{gathered}
$$

For any $R \in S O(3)$ then $3 \geq \operatorname{tr}(R) \geq-1$. If $\operatorname{tr}(R)=3$ then $\theta=0$ in angle-axis coordinates and $R=I$. If $\operatorname{tr}(R)=-1$ then $\theta= \pm \pi, R$ has real eigenvalues $(1,-1,-1)$, and there exists an orthogonal diagonalising transformation $U \in S O(3)$ such that $U R U^{T}=\operatorname{diag}(1,-1,-1)$.

For any two signals $x(t): \mathbb{R} \rightarrow M_{x}, y(t): \mathbb{R} \rightarrow M_{y}$ are termed asymptotically dependent if there exists a nondegenerate function $f_{t}: M_{x} \times M_{y} \rightarrow \mathbb{R}$ and a time $T$ such that for any $t>T$

$$
f_{t}(x(t), y(t))=0 .
$$

By the term non-degenerate we mean that the Hessian of $f_{t}$ at any point $(x, y)$ is full rank. The two signals are termed asymptotically independent if for any non-degenerate $f_{t}$ and any $T$ there exists $t_{1}>T$ with $f_{t}\left(x\left(t_{1}\right), y\left(t_{1}\right)\right) \neq 0$.

\section{B. Measurements}

The measurements available from a typical inertial measurement unit are 3-axis rate gyros, 3-axis accelerometers and 3 -axis magnetometers. The reference frame of the strap down IMU is termed the body-fixed-frame $\{B\}$. The inertial frame is denoted $\{A\}$. The rotation $R={ }_{B}^{A} R$ denotes the relative orientation of $\{B\}$ with respect to $\{A\}$.

Rate Gyros: The rate gyro measures angular velocity of $\{B\}$ relative to $\{A\}$ expressed in the body-fixed-frame of reference $\{B\}$. The error model used in this paper is

$$
\Omega^{y}=\Omega+b+\mu \in \mathbb{R}^{3}
$$

where $\Omega \in\{B\}$ denotes the true value, $\mu$ denotes additive measurement noise and $b$ denotes a constant (or slowly time-varying) gyro bias.
Accelerometer: Denote the instantaneous linear acceleration of $\{B\}$ relative to $\{A\}$, expressed in $\{A\}$, by $\dot{v}$. An ideal accelerometer, 'strapped down' to the body-fixed-frame $\{B\}$, measures the instantaneous linear acceleration of $\{B\}$ minus the (conservative) gravitational acceleration field $g_{0}$ (where we consider $g_{0}$ expressed in the inertial frame $\{A\})$, and provides a measurement expressed in the body-fixed-frame $\{B\}$. In practice, the output $a$ from a MEMS component accelerometer has added bias and noise,

$$
a=R^{T}\left(\dot{v}-g_{0}\right)+b_{a}+\mu_{a},
$$

where $b_{a}$ is a bias term and $\mu_{a}$ denotes additive measurement noise. Normally, the gravitational field $g_{0}=\left|g_{0}\right| e_{3}$ where $\left|g_{0}\right| \approx 9.8$ dominates the value of $a$ for low frequency response. Thus, it is common to use

$$
v_{a}=\frac{a}{|a|} \approx-R^{T} e_{3}
$$

as a low-frequency estimate of the inertial $z$-axis expressed in the body-fixed-frame.

Magnetometer: The magnetometers provide measurements of the magnetic field

$$
m=R^{T A} m+B_{m}+\mu_{b}
$$

where ${ }^{A} m$ is the Earths magnetic field (expressed in the inertial frame), $B_{m}$ is a body-fixed-frame expression for the local magnetic disturbance and $\mu_{b}$ denotes measurement noise. The noise $\mu_{b}$ is usually quite low for magnetometer readings, however, the local magnetic disturbance can be very significant, especially if the IMU is strapped down to an MAV with electric motors. Only the direction of the magnetometer output is relevant for attitude estimation and we will use a vectorial measurement

$$
v_{m}=\frac{m}{|m|}
$$

in the following development

The measured vectors $v_{a}$ and $v_{m}$ can be used to construct an instantaneous algebraic measurement of the rotation ${ }_{B}^{A} R$ : $\{B\} \rightarrow\{A\}$

$R_{y}=\arg \min _{R \in S O(3)}\left(\lambda_{1}\left|e_{3}-R v_{a}\right|^{2}+\lambda_{2}\left|v_{m}^{*}-R v_{m}\right|^{2}\right) \approx{ }_{B}^{A} R$

where $v_{m}^{*}$ is the inertial direction of the magnetic field in the locality where data is acquired. The weights $\lambda_{1}$ and $\lambda_{2}$ are chosen depending on the relative confidence in the sensor outputs. Due to the computational complexity of solving an optimisation problem the reconstructed rotation is often obtained in a suboptimal manner where the constraints are applied in sequence; that is, two degrees of freedom in the rotation matrix are resolved using the acceleration readings and the final degree of freedom is resolved using the magnetometer. As a consequence, the error properties of the reconstructed attitude $R_{y}$ can be difficult to characterise. Moreover, if either magnetometer or accelerometer readings are unavailable (due to local magnetic disturbance or high acceleration manoeuvres) then it is impossible to resolve the vectorial measurements into a unique instantaneous algebraic measurement of attitude. 


\section{Error criteria for estimation on $S O(3)$}

Let $\hat{R}$ denote an estimate of the body-fixed rotation matrix $R={ }_{B}^{A} R$. The rotation $\hat{R}$ can be considered as coordinates for the estimator frame of reference $\{E\}$. It is also associated with the frame transformation

$$
\hat{R}={ }_{E}^{A} \hat{R}:\{E\} \rightarrow\{A\} .
$$

The goal of attitude estimate is to drive $\hat{R} \rightarrow R$. The estimation error used is the relative rotation from body-fixedframe $\{B\}$ to the estimator frame $\{E\}$

$$
\tilde{R}:=\hat{R}^{T} R, \quad \tilde{R}={ }_{B}^{E} \tilde{R}:\{B\} \rightarrow\{E\} .
$$

The proposed observer design is based on Lyapunov stability analysis. The Lyapunov functions used are inspired by the cost function

$$
\begin{aligned}
E_{\text {tr }} & :=\frac{1}{4}\left\|I_{3}-\tilde{R}\right\|^{2}=\frac{1}{4} \operatorname{tr}\left(\left(I_{3}-\tilde{R}\right)^{T}\left(I_{3}-\tilde{R}\right)\right) \\
& =\frac{1}{2} \operatorname{tr}\left(I_{3}-\tilde{R}\right)
\end{aligned}
$$

One has that

$$
E_{\mathrm{tr}}=\frac{1}{2} \operatorname{tr}(I-\tilde{R})=(1-\cos (\theta))=2 \sin (\theta / 2)^{2} .
$$

where $\theta$ is the angle associated with the rotation from $\{B\}$ to frame $\{E\}$. Thus, driving Eq. 2 to zero ensures that $\theta \rightarrow 0$.

\section{COMPlementary FILTERS ON $S O(3)$}

In this section, a general framework for non-linear complementary filtering on the special orthogonal group is introduced. The theory is first developed for the idealised case where $R(t)$ and $\Omega(t)$ are assumed to be known and used to drive the filter dynamics. Filter design for real world signals is considered in later sections.

The goal of attitude estimation is to provide a set of dynamics for an estimate $\hat{R}(t) \in S O(3)$ to drive the error rotation (Eq. 1) $\tilde{R}(t) \rightarrow I_{3}$. The kinematics of the true system are

$$
\dot{R}=R \Omega_{\times}=(R \Omega)_{\times} R
$$

where $\Omega \in\{B\}$. The proposed observer equation is posed directly as a kinematic system for an attitude estimate $\hat{R}$ on $S O(3)$. The observer kinematics include a prediction term based on the $\Omega$ measurement and an innovation or correction term $\omega:=\omega(\tilde{R})$ derived from the error $\tilde{R}$. The general form proposed for the observer is

$$
\dot{\hat{R}}=\left(R \Omega+k_{P} \hat{R} \omega\right)_{\times} \hat{R}, \quad \hat{R}(0)=\hat{R}_{0},
$$

where $k_{P}>0$ is a positive gain. The term $\left(R \Omega+k_{P} \hat{R} \omega\right) \in$ $\{A\}$ is expressed in the inertial frame. The body-fixed-frame angular velocity is mapped back into the inertial frame ${ }^{A} \Omega=$ $R \Omega$. If no correction term is used $\left(k_{P} \omega \equiv 0\right)$ then the error rotation $\tilde{R}$ is constant,

$$
\begin{aligned}
\dot{\tilde{R}} & =\hat{R}^{T}(R \Omega)_{\times}^{T} R+\hat{R}^{T}(R \Omega)_{\times} R \\
& =\hat{R}^{T}\left(-(R \Omega)_{\times}+(R \Omega)_{\times}\right) R=0 .
\end{aligned}
$$

The correction term $\omega:=\omega(\tilde{R}) \in\{E\}$ is considered to be in the estimator frame of reference. It can be thought of as a non-linear approximation of the error between $R$ and $\hat{R}$ as measured from the frame of reference associated with $\hat{R}$. In practice, it will be implemented as an error between a measured estimate $R_{y}$ of $R$ and the estimate $\hat{R}$.

The goal of the observer design is to find a simple expression for $\omega$ that leads to robust convergence of $\tilde{R} \rightarrow I$. In prior work [35], [36] the authors introduced the following correction term

$$
\omega:=\operatorname{vex}\left(\mathbb{P}_{a}(\tilde{R})\right)=\operatorname{vex}\left(\mathbb{P}_{a}\left(\hat{R}^{T} R_{y}\right)\right)
$$

This choice leads to an elegant Lyapunov analysis of the filter stability. Differentiating the storage function Eq. 2 along trajectories of Eq. 5 yields

$$
\begin{aligned}
\dot{E}_{\mathrm{tr}}= & -\frac{1}{2} \operatorname{tr}(\dot{\tilde{R}})=-\frac{k_{P}}{2} \operatorname{tr}\left(\omega_{\times}^{T} \tilde{R}\right) \\
& =-\frac{k_{P}}{2} \operatorname{tr}\left[\omega_{\times}^{T}\left(\mathbb{P}_{s}(\tilde{R})+\mathbb{P}_{a}(\tilde{R})\right)\right]=-\frac{k_{P}}{2} \operatorname{tr}\left[\omega_{\times}^{T} \mathbb{P}_{a}(\tilde{R})\right] \\
& =-\frac{k_{P}}{2}\left\langle\left\langle\omega_{\times}, \mathbb{P}_{a}(\tilde{R})\right\rangle\right\rangle=-k_{P}|\omega|^{2}
\end{aligned}
$$

In Mahony et al. [35] a local stability analysis of the filter dynamics Eq. 5 is provided based on this derivation. In Section IV a global stability analysis for these dynamics is provided.

We term the filter Eq. 5 a complementary filter on $S O(3)$ since it recaptures the block diagram structure of a classical complementary filter (cf. Appendix A). In Figure 2: The ' $\hat{R}^{T}$,

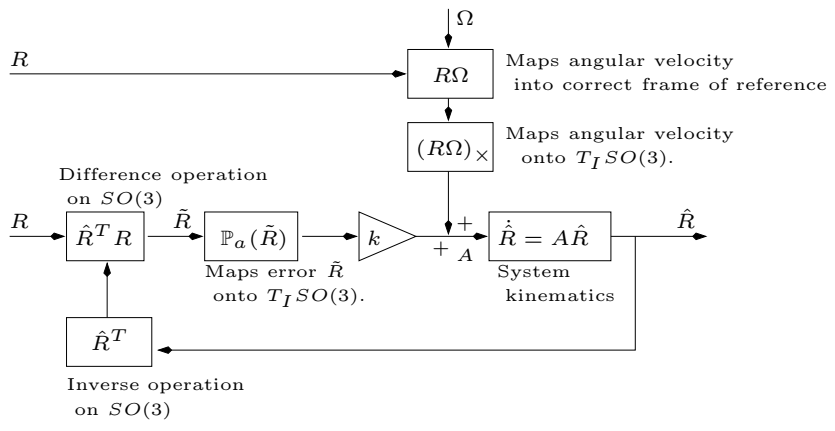

Fig. 2. Block diagram of the general form of a complementary filter on $S O(3)$.

operation is an inverse operation on $S O(3)$ and is equivalent to a '-' operation for a linear complementary filter. The ' $\hat{R}^{T} R_{y}$ ' operation is equivalent to generating the error term ' $y-\hat{x}$ '. The two operations $\mathbb{P}_{a}(\tilde{R})$ and $(R \Omega)_{\times}$are maps from error space and velocity space into the tangent space of $S O(3)$; operations that are unnecessary on Euclidean space due to the identification $T_{x} \mathbb{R}^{n} \equiv \mathbb{R}^{n}$. The kinematic model is the Liegroup equivalent of a first order integrator.

To implement the complementary filter it is necessary to map the body-fixed-frame velocity $\Omega$ into the inertial frame. In practice, the 'true' rotation $R$ is not available and an estimate of the rotation must be used. Two possibilities are considered: direct complementary filter: The constructed attitude $R_{y}$ is used to map the velocity into the inertial frame

$$
\dot{\hat{R}}=\left(R_{y} \Omega_{y}+k_{P} \hat{R} \omega\right)_{\times} \hat{R} .
$$

A block diagram of this filter design is shown in Figure 3. This approach can be linked to observers documented 
in earlier work [30], [32] (cf. Appendix B). The approach has the advantage that it does not introduce an additional feedback loop in the filter dynamics, however, high frequency noise in the reconstructed attitude $R_{y}$ will enter into the feed-forward term of the filter.

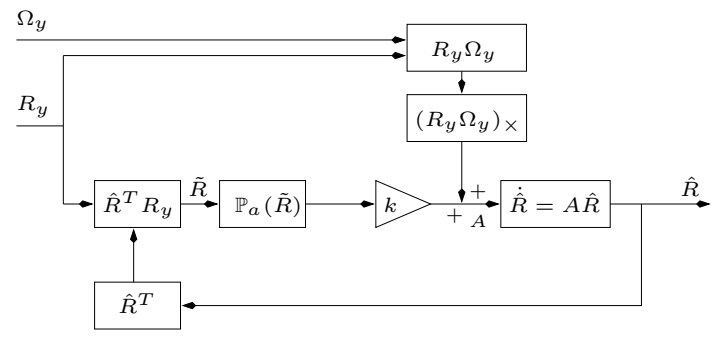

Fig. 3. Block diagram of the direct complementary filter on $S O(3)$.

passive complementary filter: The filtered attitude $\hat{R}$ is used in the predictive velocity term

$$
\dot{\hat{R}}=\left(\hat{R} \Omega_{y}+k_{P} \hat{R} \omega\right)_{\times} \hat{R} .
$$

A block diagram of this architecture is shown in Figure 4. The advantage lies in avoiding corrupting the predictive angular velocity term with the noise in the reconstructed pose. However, the approach introduces a secondary feedback loop in the filter and stability needs to be proved.

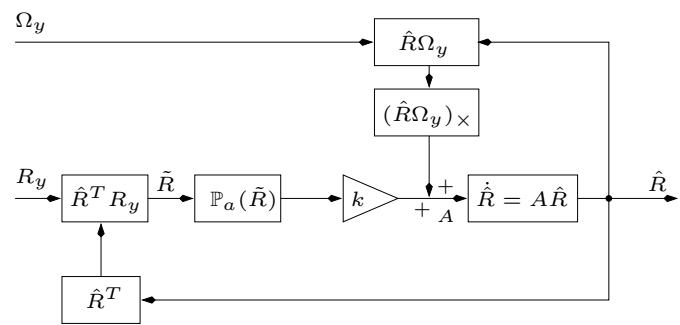

Fig. 4. Block diagram of the passive complementary filter on $S O(3)$.

A key observation is that the Lyapunov stability analysis in Eq. 8 is still valid for Eq. 9, since

$$
\begin{aligned}
\dot{E}_{\mathrm{tr}}= & -\frac{1}{2} \operatorname{tr}(\dot{\tilde{R}})=-\frac{1}{2} \operatorname{tr}\left(-\left(\Omega+k_{P} \omega\right)_{\times} \tilde{R}+\tilde{R} \Omega_{\times}\right) \\
& =-\frac{1}{2} \operatorname{tr}\left(\left[\tilde{R}, \Omega_{\times}\right]\right)-\frac{k_{P}}{2} \operatorname{tr}\left(\omega_{\times}^{T} \tilde{R}\right)=-k_{P}|\omega|^{2},
\end{aligned}
$$

using the fact that the trace of a commutator is zero, $\operatorname{tr}\left(\left[\tilde{R}, \Omega_{\times}\right]\right)=0$. The filter is termed a passive complimentary filter since the cross coupling between $\Omega$ and $\tilde{R}$ does not contribute to the derivative of the Lyapunov function. A global stability analysis is provided in Section IV.

There is no particular theoretical advantage to either the direct or the passive filter architecture in the case where exact measurements are assumed. However, it is straightforward to see that the passive filter (Eq. 9) can be written

$$
\dot{\hat{R}}=\hat{R}\left(\Omega_{\times}+k_{P} \mathbb{P}_{a}(\tilde{R})\right) .
$$

This formulation suppresses entirely the requirement to represent $\Omega$ and $\omega=k_{P} \mathbb{P}_{a}(\tilde{R})$ in the inertial frame and leads to the architecture shown in Figure 5. The passive complementary filter avoids coupling the reconstructed attitude noise into the predictive velocity term of the observer, has a strong Lyapunov stability analysis, and provides a simple and elegant realisation that will lead to the results in Section V.

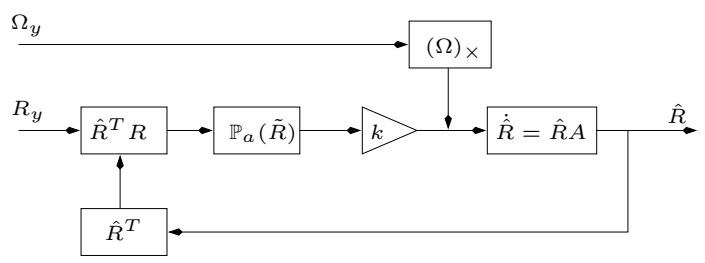

Fig. 5. Block diagram of the simplified form of the passive complementary filter.

\section{Stability ANALysis}

In this section, the direct and passive complementary filters on $S O(3)$ are extended to provide on-line estimation of timevarying bias terms in the gyroscope measurements and global stability results are derived. Preliminary results were published in [35], [36].

For the following work it is assumed that a reconstructed rotation $R_{y}$ and a biased measure of angular velocity $\Omega^{y}$ are available

$$
\begin{array}{lr}
R_{y} \approx R, & \text { valid for low frequencies, } \\
\Omega_{y} \approx \Omega+b & \text { for constant bias } b .
\end{array}
$$

The approach taken is to add an integrator to the compensator term in the feedback equation of the complementary filter.

Let $k_{P}, k_{I}>0$ be positive gains and define

Direct complementary filter with bias correction:

$$
\begin{array}{lrl}
\dot{\hat{R}}=\left(R_{y}\left(\Omega^{y}-\hat{b}\right)+k_{P} \hat{R} \omega\right)_{\times} \hat{R}, & \hat{R}(0)=\hat{R}_{0}, \\
\dot{\hat{b}}=-k_{I} \omega, & \hat{b}(0)=\hat{b}_{0}, \\
\omega=\operatorname{vex}\left(\mathbb{P}_{a}(\tilde{R})\right), & \tilde{R}=\hat{R}^{T} R_{y} .
\end{array}
$$

Passive complementary filter with bias correction:

$$
\begin{aligned}
\dot{\hat{R}} & =\hat{R}\left(\Omega^{y}-\hat{b}+k_{P} \omega\right)_{\times}, & & \hat{R}(0)=\hat{R}_{0}, \\
\dot{\hat{b}} & =-k_{I} \omega, & \hat{b}(0) & =\hat{b}_{0}, \\
\omega & =\operatorname{vex}\left(\mathbb{P}_{a}(\tilde{R})\right), & & \tilde{R}=\hat{R}^{T} R_{y} .
\end{aligned}
$$

The non-linear stability analysis is based on the idea of an adaptive estimate for the unknown bias value.

Theorem 4.1: [Direct complementary filter with bias correction.] Consider the rotation kinematics Eq. 4 for a time-varying $R(t) \in S O(3)$ and with measurements given by Eq. 11. Let $(\hat{R}(t), \hat{b}(t))$ denote the solution of Eq. 12 . Define error variables $\tilde{R}=\hat{R}^{T} R$ and $\tilde{b}=b-\hat{b}$. Define $\mathbb{U} \subseteq S O(3) \times \mathbb{R}^{3}$ by

$$
\mathbb{U}=\left\{(\tilde{R}, \tilde{b}) \mid \operatorname{tr}(\tilde{R})=-1, \mathbb{P}_{a}\left(\tilde{b}_{\times} \tilde{R}\right)=0\right\} .
$$

Then: 
1) The set $\mathbb{U}$ is forward invariant and unstable with respect to the dynamic system Eq. 12.

2) The error $(\tilde{R}(t), \tilde{b}(t))$ is locally exponentially stable to $(I, 0)$.

3) For almost all initial conditions $\left(\tilde{R}_{0}, \tilde{b}_{0}\right) \notin \mathbb{U}$ the trajectory $(\hat{R}(t), \hat{b}(t))$ converges to the trajectory $(R(t), b)$.

Proof: Substituting for the error model (Eq. 11), Equation 12a becomes

$$
\dot{\hat{R}}=\left(R(\Omega+\tilde{b})+k_{P} \hat{R} \omega\right)_{\times} \hat{R} .
$$

Differentiating $\tilde{R}$ it is straightforward to verify that

$$
\dot{\tilde{R}}=-k_{P} \omega_{\times} \tilde{R}-\tilde{b}_{\times} \tilde{R}
$$

Define a candidate Lyapunov function by

$$
V=\frac{1}{2} \operatorname{tr}\left(I_{3}-\tilde{R}\right)+\frac{1}{2 k_{I}}|\tilde{b}|^{2}=E_{\text {tr }}+\frac{1}{2 k_{I}}|\tilde{b}|^{2}
$$

Differentiating $V$ one obtains

$$
\begin{aligned}
\dot{V}= & -\frac{1}{2} \operatorname{tr}(\dot{\tilde{R}})-\frac{1}{k_{I}} \tilde{b}^{T} \dot{\hat{b}} \\
= & \frac{1}{2} \operatorname{tr}\left(k_{P} \omega_{\times} \tilde{R}+\tilde{b}_{\times} \tilde{R}\right)-\frac{1}{k_{I}}\langle\tilde{b}, \dot{\hat{b}}\rangle \\
= & \frac{-k_{P}}{2}\left\langle\left\langle\omega_{\times}, \mathbb{P}_{a}(\tilde{R})+\mathbb{P}_{s}(\tilde{R})\right\rangle\right\rangle \\
& \quad-\frac{1}{2}\left\langle\left\langle\tilde{b}_{\times}, \mathbb{P}_{a}(\tilde{R})+\mathbb{P}_{s}(\tilde{R})\right\rangle\right\rangle-\frac{1}{k_{I}}\langle\tilde{b}, \dot{\hat{b}}\rangle \\
& =-k_{P}\left\langle\omega, \operatorname{vex}\left(\mathbb{P}_{a}(\tilde{R})\right)\right\rangle-\left\langle\tilde{b}, \operatorname{vex}\left(\mathbb{P}_{a}(\tilde{R})\right\rangle-\frac{1}{k_{I}}\langle\tilde{b}, \dot{\hat{b}}\rangle\right.
\end{aligned}
$$

Substituting for $\dot{\hat{b}}$ and $\omega$ (Eqn's 12b and 12c) one obtains

$$
\dot{V}=-k_{P}|\omega|^{2}=-k_{P}\left|\operatorname{vex} \mathbb{P}_{a}(\tilde{R})\right|^{2}
$$

Lyapunov's direct method ensures that $\omega$ converges asymptotically to zero [40]. Recalling that $\left\|\mathbb{P}_{a}(\tilde{R})\right\|=\sqrt{2} \sin (\theta)$, where $(\theta, a)$ denotes the angle-axis coordinates of $\tilde{R}$. It follows that $\omega \equiv 0$ implies either $\tilde{R}=I$, or $\log (\tilde{R})=\pi a_{\times}$for $|a|=1$. In the second case one has the condition $\operatorname{tr}(\tilde{R})=-1$. Note that $\omega=0$ is also equivalent to requiring $\tilde{R}=\tilde{R}^{T}$ to be symmetric.

It is easily verified that $(I, 0)$ is an isolated equilibrium of the error dynamics Eq. 18.

From the definition of $\mathbb{U}$ one has that $\omega \equiv 0$ on $\mathbb{U}$. We will prove that $\mathbb{U}$ is forward invariant under the filter dynamics Eqn's 12. Setting $\omega=0$ in Eq. 15 and Eq. 12 b yields

$$
\dot{\tilde{R}}=-\tilde{b}_{\times} \tilde{R}, \quad \dot{\hat{b}}=0 .
$$

For initial conditions $\left(\tilde{R}_{0}, \tilde{b}_{0}\right)=\left(\tilde{R}_{0}, \tilde{b}_{0}\right) \in \mathbb{U}$ the solution of Eq. 18 is given by

$$
\tilde{R}(t)=\exp \left(-t \tilde{b}_{\times}\right) \tilde{R}_{0}, \quad \tilde{b}(t)=\tilde{b}_{0}, \quad\left(\tilde{R}_{0}, \tilde{b}_{0}\right) \in \mathbb{U} .
$$

We verify that Eq. 19 is also a general solution of Eqn's 15 and $12 \mathrm{~b}$. Differentiating $\operatorname{tr}(\tilde{R})$ yields

$$
\begin{aligned}
\frac{d}{d t} \operatorname{tr}(\tilde{R}) & =-\operatorname{tr}\left(\tilde{b}_{\times} \exp \left(-t \tilde{b}_{\times}\right) \tilde{R}_{0}\right) \\
& =\operatorname{tr}\left(\exp \left(-t \tilde{b}_{\times}\right) \frac{\left(\tilde{b}_{\times} \tilde{R}_{0}+\tilde{R}_{0} \tilde{b}_{\times}\right)}{2}\right) \\
& =\operatorname{tr}\left(\exp \left(-t \tilde{b}_{\times}\right) \mathbb{P}_{a}\left(\tilde{b}_{\times} \tilde{R}_{0}\right)\right)=0
\end{aligned}
$$

where the second line follows since $\tilde{b}_{\times}$commutes with $\exp \left(\tilde{b}_{\times}\right)$and the final equality is due to the fact that $\mathbb{P}_{a}\left(b_{\times} \tilde{R}_{0}\right)=0$, a consequence of the choice of initial conditions $\left(\tilde{R}_{0}, \tilde{b}_{0}\right) \in \mathbb{U}$. It follows that $\operatorname{tr}(\tilde{R}(t))=-1$ on solution of Eq. 19 and hence $\omega \equiv 0$. Classical uniqueness results verify that Eq. 19 is a solution of Eqn's 15 and 12b. It remains to show that such solutions remain in $\mathbb{U}$ for all time. The condition on $\tilde{R}$ is proved above. To see that $\mathbb{P}_{a}\left(\tilde{b}_{\times} \tilde{R}\right) \equiv 0$ we compute

$$
\frac{d}{d t} \mathbb{P}_{a}\left(\tilde{b}_{\times} \tilde{R}\right)=-\mathbb{P}_{a}\left(\tilde{b}_{\times}^{2} \tilde{R}\right)=-\mathbb{P}_{a}\left(\tilde{b}_{\times} \tilde{R} \tilde{b}_{\times}^{T}\right)=0
$$

as $\tilde{R}=\tilde{R}^{T}$. This proves that $\mathbb{U}$ is forward invariant.

Applying LaSalle's principle to the solutions of Eq. 12 it follows that either $(\tilde{R}, \tilde{b}) \rightarrow(I, 0)$ asymptotically or $(\tilde{R}, \tilde{b}) \rightarrow$ $\left(\tilde{R}_{*}(t), \tilde{b}_{0}\right)$ where $\left(\tilde{R}_{*}(t), \tilde{b}_{0}\right) \in \mathbb{U}$ is a solution of Eq. 18.

To determine the local stability properties of the invariant sets we compute the linearisation of the error dynamics. We will prove exponential stability of the isolated equilibrium point $(I, 0)$ first and then return to prove instability of the set $\mathbb{U}$. Define $x, y \in \mathbb{R}^{3}$ as the first order approximations of $\tilde{R}$ and $\tilde{b}$ around $(I, 0)$

$$
\begin{aligned}
\tilde{R} & \approx\left(I+x_{\times}\right), \quad x_{\times} \in \mathfrak{s o}(3) \\
\tilde{b} & =-y .
\end{aligned}
$$

The sign change in Eq. 20b simplifies the analysis of the linearisation. Substituting into Eq. 15, computing $\dot{\tilde{b}}$ and discarding all terms of quadratic or higher order in $(x, y)$ yields

$$
\frac{d}{d t}\left(\begin{array}{l}
x \\
y
\end{array}\right)=\left(\begin{array}{cc}
-k_{P} I_{3} & I_{3} \\
-k_{I} I_{3} & 0
\end{array}\right)\left(\begin{array}{l}
x \\
y
\end{array}\right)
$$

For positive gains $k_{P}, k_{I}>0$ the linearised error system is strictly stable. This proves part ii) of the theorem statement.

To prove that $\mathbb{U}$ is unstable, we use the quaternion formulation (see Appendix B). Using Eq. 49, the error dynamics of the quaternion $\tilde{q}=(\tilde{s}, \tilde{v})$ associated to the rotation $\tilde{R}$ is given by

$$
\begin{aligned}
\dot{\tilde{s}} & =\frac{1}{2}\left(k_{P} \tilde{s}|\tilde{v}|^{2}+\tilde{v}^{T} \tilde{b}\right), \\
\dot{\tilde{b}} & =k_{I} \tilde{s} \tilde{v} \\
\dot{\tilde{v}} & =-\frac{1}{2}\left(\tilde{s}\left(\tilde{b}+k_{P} \tilde{s} \tilde{v}\right)+\tilde{b} \times \tilde{v}\right),
\end{aligned}
$$

It is straightforward to verify that the invariant set associated to the error dynamics is characterised by

$$
\mathbb{U}=\left\{(\tilde{s}, \tilde{v}, \tilde{b})|\tilde{s}=0,| \tilde{v} \mid=1, \tilde{b}^{T} \tilde{v}=0\right\}
$$


Define $y=\tilde{b}^{T} \tilde{v}$, then an equivalent characterisation of $\mathbb{U}$ is given by $(\tilde{s}, y)=(0,0)$. We study the stability properties of the equilibrium $(0,0)$ of $(\tilde{s}, y)$ evolving under the filter dynamics Eq. 12. Combining Eq. 22c and 22b, one obtains the following dynamics for $\dot{y}$

$$
\begin{aligned}
\dot{y} & =\tilde{v}^{T} \dot{\tilde{b}}+\tilde{b}^{T} \dot{\tilde{v}} \\
& =k_{I} \tilde{s}|\tilde{v}|^{2}-\frac{1}{2} \tilde{s}|\tilde{b}|^{2}-\frac{1}{2} k_{P} \tilde{s}^{2} y
\end{aligned}
$$

Linearising around small values of $(\tilde{s}, y)$ one obtains

$$
\left(\begin{array}{c}
\dot{\tilde{s}} \\
\dot{y}
\end{array}\right)=\left(\begin{array}{cc}
\frac{1}{2} k_{P} & \frac{1}{2} \\
k_{I}-\frac{1}{2}\left|\tilde{b}_{0}\right|^{2} & 0
\end{array}\right)\left(\begin{array}{c}
\tilde{s} \\
y
\end{array}\right)
$$

Since $K_{P}$ and $K_{I}$ are positive gains it follows that the linearisation is unstable around the point $(0,0)$ and this completes the proof of part i).

The linearisation of the dynamics around the unstable set is either strongly unstable (for large values of $\left|\tilde{b}_{0}\right|^{2}$ ) or hyperbolic (both positive and negative eigenvalues). Since $\tilde{b}_{0}$ depends on the initial condition then there there will be trajectories that converge to $\mathbb{U}$ along the stable centre manifold [40] associated with the stable direction of the linearisation. From classical centre manifold theory it is known that such trajectories are measure zero in the overall space. Observing in addition that $\mathbb{U}$ is measure zero in $S O(3) \times \mathbb{R}^{3}$ proves part iii) and the full proof is complete.

The direct complimentary filter is closely related to quaternion based attitude filters published over the last fifteen years [9], [30], [32]. Details of the similarities and differences is given in Appendix B where we present quaternion versions of the filters we propose in this paper. Apart from the formulation directly on $S O(3)$, the present paper extends earlier work by proposing globally defined observer dynamics and a full global analysis. To the authors best understanding, all prior published algorithms depend on a $\operatorname{sgn}(\theta)$ term that is discontinuous on $\mathbb{U}$ (Eq. 14). Given that the observers are not well defined on the set $\mathbb{U}$ the analysis for prior work is necessarily non-global. However, having noted this, the recent work of Thienel et al. [30] provides an elegant powerful analysis that transforms the observer error dynamics into a linear timevarying system (the transformation is only valid on a domain on $S O(3) \times \mathbb{R}^{3}-\mathbb{U}$ ) for which global asymptotic stability is proved. This analysis provides a global exponential stability under the assumption that the observer error trajectory does not intersect $\mathbb{U}$. In all practical situations the two approaches are equivalent.

The remainder of the section is devoted to proving an analogous result to Theorem 4.1 for the passive complementary filter dynamics. In this case, it is necessary to deal with non-autonomous terms in the error dynamics due to passive coupling of the driving term $\Omega$ into the filter error dynamics. Interestingly, the non-autonomous term acts in our favour to disturb the forward invariance properties of the set $\mathbb{U}$ (Eq. 14) and reduce the size of the unstable invariant set.

Theorem 4.2: [Passive complementary filter with bias correction.] Consider the rotation kinematics Eq. 4 for a time-varying $R(t) \in S O(3)$ and with measurements given by Eq. 11. Let $(\hat{R}(t), \hat{b}(t))$ denote the solution of Eq. 13. Define error variables $\tilde{R}=\hat{R}^{T} R$ and $\tilde{b}=b-\hat{b}$. Assume that $\Omega(t)$ is a bounded, absolutely continuous signal and that the pair of signals $(\Omega(t), \tilde{R})$ are asymptotically independent (see $\S \mathrm{II}-\mathrm{A}$ ). Define $\mathbb{U}_{0} \subseteq S O(3) \times \mathbb{R}^{3}$ by

$$
\mathbb{U}_{0}=\{(\tilde{R}, \tilde{b}) \mid \operatorname{tr}(\tilde{R})=-1, \tilde{b}=0\} .
$$

Then:

1) The set $\mathbb{U}_{0}$ is forward invariant and unstable with respect to the dynamic system 13 .

2) The error $(\tilde{R}(t), \tilde{b}(t))$ is locally exponentially stable to $(I, 0)$.

3) For almost all initial conditions $\left(\tilde{R}_{0}, \tilde{b}_{0}\right) \notin \mathbb{U}_{0}$ the trajectory $(\hat{R}(t), \hat{b}(t))$ converges to the trajectory $(R(t), b)$.

Proof: Substituting for the error model (Eq. 11) in Eqn's 13 and differentiating $\tilde{R}$, it is straightforward to verify that

$$
\begin{aligned}
\dot{\tilde{R}} & =\left[\tilde{R}, \Omega_{\times}\right]-k_{P} \omega_{\times} \tilde{R}-\tilde{b}_{\times} \tilde{R}, \\
\dot{\tilde{b}} & =k_{I} \omega
\end{aligned}
$$

The proof proceeds by differentiating the Lyapunov-like function Eq. 16 for solutions of Eq. 13. Following an analogous derivation to that in Theorem 4.1, but additionally exploiting the cancellation $\operatorname{tr}\left(\left[\tilde{R}, \Omega_{\times}\right]\right)=0$, it may be verified that

$$
\dot{V}=-k_{P}|\omega|^{2}=-k_{P}\left|\operatorname{vex}\left(\mathbb{P}_{a}(\tilde{R})\right)\right|^{2}
$$

where $V$ is given by Eq. 16 . This bounds $V(t) \leq V(0)$, and it follows $\tilde{b}$ is bounded. LaSalle's principle cannot be applied directly since the dynamics Eq. 24a are not autonomous. The function $\dot{V}$ is uniformly continuous since the derivative

$$
\ddot{V}=-k_{P} \mathbb{P}_{a}(\tilde{R})^{T}\left(\mathbb{P}_{a}\left(\left[\tilde{R}, \Omega_{\times}\right]\right)-\mathbb{P}_{a}\left(\left(k_{P} \omega-\tilde{b}\right)_{\times}\right) \tilde{R}\right)
$$

is uniformly bounded. Applying Barbalat's lemma proves asymptotic convergence of $\omega=\operatorname{vex}\left(\mathbb{P}_{a}(\tilde{R})\right)$ to zero.

Direct substitution shows that $(\tilde{R}, \tilde{b})=(I, 0)$ is an equilibrium point of Eq. 24. Note that $\mathbb{U}_{0} \subset \mathbb{U}$ (Eq. 14) and hence $\omega \equiv 0$ on $\mathbb{U}$ (Th. 4.1). For $(\tilde{R}, \tilde{b}) \in \mathbb{U}_{0}$ the error dynamics Eq. 24 become

$$
\dot{\tilde{R}}=\left[\tilde{R}, \Omega_{\times}\right], \quad \dot{\tilde{b}}=0 .
$$

The solution of this ordinary differential equation is given by

$$
\tilde{R}(t)=\exp (-A(t)) \tilde{R}_{0} \exp (A(t)), \quad A(t)=\int_{0}^{t} \Omega_{\times} d \tau .
$$

Since $A(t)$ is anti-symmetric for all time then $\exp (-A(t))$ is orthogonal and since $\exp (-A(t))=\exp (A(t))^{T}$ it follows $\tilde{R}$ is symmetric for all time. It follows that $\mathbb{U}_{0}$ is forward invariant under the filter dynamics Eq. 13. We prove by contradiction that $\mathbb{U}_{0} \subset \mathbb{U}$ is the largest forward invariant set of the closedloop dynamics Eq. 13 such that $\omega \equiv 0$. Assume that there exits $\left(\tilde{R}_{0}, \tilde{b}_{0}\right) \in \mathbb{U}-\mathbb{U}_{0}$ such that $(\tilde{R}(t), \tilde{b}(t))$ remains in $\mathbb{U}$ for all time. One has that $\mathbb{P}_{a}\left(\tilde{b}_{\times} \tilde{R}\right)=0$ on this trajectory. Consequently,

$$
\begin{aligned}
\frac{d}{d t} \mathbb{P}_{a}\left(\tilde{b}_{\times} \tilde{R}\right) & =\mathbb{P}_{a}\left(\tilde{b}_{\times}\left[\tilde{R}, \Omega_{\times}\right]\right)-\mathbb{P}\left(\tilde{b}_{\times} \tilde{R} b_{\times}^{T}\right) \\
& =\mathbb{P}_{a}\left(\tilde{b}_{\times}\left[\tilde{R}, \Omega_{\times}\right]\right) \\
& =-\frac{1}{2}\left((\tilde{b} \times \Omega)_{\times} \tilde{R}+\tilde{R}(\tilde{b} \times \Omega)_{\times}\right)=0,
\end{aligned}
$$


where we have used

$$
2 \mathbb{P}_{a}\left(\tilde{b}_{\times} \tilde{R}\right)=\tilde{b}_{\times} \tilde{R}+\tilde{R} \tilde{b}_{\times}=0,
$$

several times in simplifying expressions. Since $(\Omega(t), \tilde{R}(t))$ are asymptotically independent then the relationship Eq. 25 must be degenerate. This implies that there exists a time $T$ such that for all $t>T$ then $\tilde{b}(t) \equiv 0$ and contradicts the assumption.

It follows that either $(\tilde{R}, \tilde{b}) \rightarrow(I, 0)$ asymptotically or $(\tilde{R}, \tilde{b}) \rightarrow\left(\tilde{R}_{*}(t), 0\right) \in \mathbb{U}_{0}$.

Analogously to Theorem 4.1 the linearisation of the error dynamics (Eq. 24) at $(I, 0)$ is computed. Let $\tilde{R} \approx I+x_{\times}$ and $\tilde{b} \approx-y$ for $x, y \in \mathbb{R}^{3}$. The linearised dynamics are the time-varying linear system

$$
\frac{d}{d t}\left(\begin{array}{l}
x \\
y
\end{array}\right)=\left(\begin{array}{cc}
-k_{P} I_{3}-\Omega(t)_{\times} & I_{3} \\
-k_{I} I_{3} & 0
\end{array}\right)\left(\begin{array}{l}
x \\
y
\end{array}\right)
$$

Let $\left|\Omega_{\max }\right|$ denote the magnitude bound on $\Omega$ and choose

$$
\begin{gathered}
\alpha_{2}>0, \quad \alpha_{1}>\frac{\alpha_{2}\left(\left|\Omega_{\max }\right|^{2}+k_{I}\right)}{k_{P}}, \\
\frac{\alpha_{1}+k_{P} \alpha_{2}}{k_{I}}<\alpha_{3}<\frac{\alpha_{1}+k_{P} \alpha_{2}}{k_{I}}+\frac{\left|\Omega_{\max }\right| \alpha_{2}}{k_{I}}
\end{gathered}
$$

Set $P, Q$ to be matrices

$$
P=\left(\begin{array}{cc}
\alpha_{1} I_{3} & -\alpha_{2} I_{3} \\
-\alpha_{2} I_{3} & \alpha_{3} I_{3}
\end{array}\right), Q=\left(\begin{array}{cc}
k_{P} \alpha_{1}-\alpha_{2} k_{I} & -\alpha_{2}\left|\Omega_{\max }\right| \\
-\alpha_{2}\left|\Omega_{\max }\right| & \alpha_{2}
\end{array}\right)
$$

It is straightforward to verify that $P$ and $Q$ are positive definite matrices given the constraints on $\left\{\alpha_{1}, \alpha_{2}, \alpha_{3}\right\}$. Consider the cost function $W=\frac{1}{2} \xi^{T} P \xi$, with $\xi=(x, y)^{T}$. Differentiating $W$ yields

$$
\begin{aligned}
\dot{W}= & -\left(k_{P} \alpha_{1}-\alpha_{2} k_{I}\right)|x|^{2}-\alpha_{2}|y|^{2} \\
& +y^{T} x\left(\alpha_{1}+k_{P} \alpha_{2}-\alpha_{3} k_{I}\right)+\alpha_{2} y^{T}(\Omega \times x)
\end{aligned}
$$

It is straightforward to verify that

$$
\frac{d}{d t}\left(\xi^{T} P \xi\right) \leq-2(|x|,|y|) Q\left(\begin{array}{l}
|x| \\
|y|
\end{array}\right) .
$$

This proves exponential stability of the linearised system at $(I, 0)$.

The linearisation of the error dynamics on a trajectory in $\mathbb{U}_{0}$ are also time varying and it is not possible to use the argument from Theorem 4.1 to prove instability. However, note that $V\left(\tilde{R}_{*}, \tilde{b}_{*}\right)=2$ for all $\left(\tilde{R}_{*}, \tilde{b}_{*}\right) \in \mathbb{U}_{0}$. Moreover, any neighbourhood of a point $\left(\tilde{R}_{*}, \tilde{b}_{*}\right) \in \mathbb{U}_{0}$ within the set $S O(3) \times \mathbb{R}^{3}$ contains points $(\tilde{R}, \tilde{b})$ such the $V(\tilde{R}, \tilde{b})<2$. Trajectories with these initial conditions cannot converge to $\mathbb{U}_{0}$ due to the decrease condition derived earlier, and it follows that $\mathbb{U}_{0}$ is unstable. Analogous to Theorem 4.1 it is still possible that a set of measure zero initial conditions, along with very specific trajectories $\Omega(t)$, such that the resulting trajectories converge to to $\mathbb{U}_{0}$. This proves part iii) and completes the proof.

Apart from the expected conditions inherited from Theorem 4.1 the key assumption in Theorem 4.2 is the independence of $\Omega(t)$ from the error signal $\tilde{R}$. The perturbation of the passive dynamics by the independent driving term $\Omega$ provides a disturbance that ensures that the adaptive bias estimate converges to the true gyroscopes' bias, a particularly useful property in practical applications.

\section{EXPLICIT ERROR FORMULATION OF THE PASSIVE COMPLEMENTARY FILTER}

A weakness of the formulation of both the direct and passive and complementary filters is the requirement to reconstruct an estimate of the attitude, $R_{y}$, to use as the driving term for the error dynamics. The reconstruction cannot be avoided in the direct filter implementation because the reconstructed attitude is also used to map the velocity into the inertial frame. In this section, we show how the passive complementary filter may be reformulated in terms of direct measurements from the inertial unit.

Let $v_{0 i} \in\{A\}, i=1, \ldots, n$, denote a set of $n$ known inertial directions. The measurements considered are bodyfixed-frame observations of the fixed inertial directions

$$
v_{i}=R^{T} v_{0 i}+\mu_{i}, \quad v_{i} \in\{B\}
$$

where $\mu_{i}$ is a noise process. Since only the direction of the measurement is relevant to the observer we assume that $\left|v_{0 i}\right|=$ 1 and normalise all measurements to ensure $\left|v_{i}\right|=1$.

Let $\hat{R}$ be an estimate of $R$. Define

$$
\hat{v}_{i}=\hat{R}^{T} v_{0 i}
$$

to be the associated estimate of $v_{i}$. For a single direction $v_{i}$, the error considered is

$$
E_{i}=1-\cos \left(\angle v_{i}, \hat{v}_{i}\right)=1-\left\langle v_{i}, \hat{v}_{i}\right\rangle
$$

which yields

$$
E_{i}=1-\operatorname{tr}\left(\hat{R}^{T} v_{0 i} v_{0 i}^{T} R\right)=1-\operatorname{tr}\left(\tilde{R} R^{T} v_{0 i} v_{0 i}^{T} R\right)
$$

For multiple measures $v_{i}$ the following cost function is considered

$$
E_{\mathrm{mes}}=\sum_{i=1}^{n} k_{i} E_{i}=\sum_{i=1}^{n} k_{i}-\operatorname{tr}(\tilde{R} M), \quad k_{i}>0,
$$

where

$$
M=R^{T} M_{0} R \quad \text { with } \quad M_{0}=\sum_{i=1}^{n} k_{i} v_{0 i} v_{0 i}^{T}
$$

Assume linearly independent inertial direction $\left\{v_{0 i}\right\}$ then the matrix $M$ is positive definite $(M>0)$ if $n \geq 3$. For $n=2$ then $M$ is positive semi-definite with one eigenvalue zero. The weights $k_{i}>0$ are chosen depending on the relative confidence in the measurements $v_{i}$. For technical reasons in the proof of Theorem 5.1 we assume additionally that the weights $k_{i}$ are chosen such that $M_{0}$ has three distinct eigenvalues $\lambda_{1}>$ $\lambda_{2}>\lambda_{3}$.

Theorem 5.1: [Explicit complementary filter with bias correction.] Consider the rotation kinematics Eq. 4 for a timevarying $R(t) \in S O(3)$ and with measurements given by Eqn's 29 and $11 \mathrm{~b}$. Assume that there are two or more, $(n \geq 2)$ vectorial measurements $v_{i}$ available. Choose $k_{i}>0$ such 
that $M_{0}$ (defined by Eq. 31) has three distinct eigenvalues. Consider the filter kinematics given by

$$
\begin{aligned}
\dot{\hat{R}} & =\hat{R}\left(\left(\Omega^{y}-\hat{b}\right)_{\times}+k_{P}\left(\omega_{\text {mes }}\right)_{\times}\right), & & \hat{R}(0)=\hat{R}_{0} \\
\dot{\hat{b}} & =-k_{I} \omega_{\text {mes }} & & \\
\omega_{\text {mes }} & :=\sum_{i=1}^{n} k_{i} v_{i} \times \hat{v}_{i}, & & k_{i}>0 .
\end{aligned}
$$

and let $(\hat{R}(t), \hat{b}(t))$ denote the solution of Eqn's 32. Assume that $\Omega(t)$ is a bounded, absolutely continuous signal and that the pair of signals $\left(\Omega(t), \tilde{R}^{T}\right)$ are asymptotically independent (see $\S$ II-A). Then:

1) There are three unstable equilibria of the filter characterised by

$$
\left(\hat{R}_{* i}, \hat{b}_{* i}\right)=\left(U_{0} D_{i} U_{0}^{T} R, b\right), i=1,2,3,
$$

where $D_{1}=\operatorname{diag}(1,-1,-1), D_{2}=\operatorname{diag}(-1,1,-1)$ and $D_{3}=\operatorname{diag}(-1,-1,1)$ are diagonal matrices with entries as shown and $U_{0} \in S O(3)$ such that $M_{0}=$ $U_{0} \Lambda U_{0}^{T}$ where $\Lambda=\operatorname{diag}\left(\lambda_{1}, \lambda_{2}, \lambda_{3}\right)$ is a diagonal matrix.

2) The error $(\tilde{R}(t), \tilde{b}(t))$ is locally exponentially stable to $(I, 0)$.

3) For almost all initial conditions $\left(\tilde{R}_{0}, \tilde{b}_{0}\right) \neq\left(\hat{R}_{* i}^{T} R, b\right)$, $i=1, \ldots, 3$, the trajectory $(\hat{R}(t), \hat{b}(t))$ converges to the trajectory $(R(t), b)$.

Proof: Define a candidate Lyapunov-like function by

$$
V=\sum_{i=1}^{n} k_{i}-\operatorname{tr}(\tilde{R} M)+\frac{1}{k_{I}} \tilde{b}^{2}=E_{\mathrm{mes}}+\frac{1}{k_{I}} \tilde{b}^{2}
$$

The derivative of $V$ is given by

$$
\begin{aligned}
\dot{V}= & -\operatorname{tr}(\dot{\tilde{R}} M+\tilde{R} \dot{M})-\frac{2}{k_{I}} \tilde{b}^{T} \dot{\hat{b}} \\
& =-\operatorname{tr}\left(\left[\tilde{R} M, \Omega_{\times}\right]-\left(\tilde{b}+k_{P} \omega_{\mathrm{mes}}\right)_{\times} \tilde{R} M\right)-\frac{2}{k_{I}} \tilde{b}^{T} \dot{\hat{b}}
\end{aligned}
$$

Recalling that the trace of a commutator is zero, the derivative of the candidate Lyapunov function can be simplified to obtain $\dot{V}=k_{P} \operatorname{tr}\left(\left(\omega_{\mathrm{mes}}\right)_{\times} \mathbb{P}_{a}(\tilde{R} M)\right)+\operatorname{tr}\left(\tilde{b}_{\times}\left(\mathbb{P}_{a}(\tilde{R} M)-\frac{1}{k_{I}} \dot{\hat{b}}_{\times}\right)\right)$

Recalling the identities in Section II-A one may write $\omega_{\text {mes }}$ as

$$
\left(\omega_{\text {mes }}\right)_{\times}=\sum_{i=1}^{n} \frac{k_{i}}{2}\left(\hat{v}_{i} v_{i}^{T}-v_{i} \hat{v}_{i}^{T}\right)=\mathbb{P}_{a}(\tilde{R} M)
$$

Introducing the expressions of $\omega_{\text {mes }}$ into the time derivative of the Lyapunov-like function $V$, Eq. 33, one obtains

$$
\dot{V}=-k_{P}\left\|\mathbb{P}_{a}(\tilde{R} M)\right\|^{2} .
$$

The Lyapunov-like function derivative is negative semidefinite ensuring that $\tilde{b}$ is bounded. Analogous to the proof of Theorem 4.2, Barbalat's lemma is invoked to show that $\mathbb{P}_{a}(\tilde{R} M)$ tends to zero asymptotically. Thus, for $\dot{V}=0$ one has

$$
\tilde{R} M=M \tilde{R}^{T} .
$$

We prove next Eq. 35 implies either $\tilde{R}=I$ or $\operatorname{tr}(\tilde{R})=-1$.

Since $\tilde{R}$ is a real matrix, the eigenvalues and eigenvectors of $\tilde{R}$ verify

$$
\tilde{R}^{T} x_{k}=\lambda_{k} x_{k} \text { and } x_{k}^{H} \tilde{R}=\lambda_{k}^{H} x_{k}^{H}
$$

where $\lambda_{k}^{H}$ (for $k=1 \ldots 3$ ) represents the complex conjugate of the eigenvalue $\lambda_{k}$ and $x_{k}^{H}$ represents the Hermitian transpose of the eigenvector $x_{k}$ associated to $\lambda_{k}$. Combining Eq. 35 and Eq. 36, one obtains

$$
\begin{aligned}
x_{k}^{H} \tilde{R} M x_{k} & =\lambda_{k}^{H} x_{k}^{H} M x_{k} \\
x_{k}^{H} M \tilde{R}^{T} x_{k} & =\lambda_{k} x_{k}^{H} M x_{k}=\lambda_{k}^{H} x_{k}^{H} M x_{k}
\end{aligned}
$$

Note that for $n \geq 3, M>0$ is positive definite and $x_{k}^{H} M x_{k}>0, \forall k=\{1,2,3\}$. One has $\lambda_{k}=\lambda_{k}^{H}$ for all $k$. In the case when $n=2$, it is simple to verify that two of the three eigenvalues are real. It follows that all three eigenvalues of $\tilde{R}$ are real since complex eigenvalues must come in complex conjugate pairs. The eigenvalues of an orthogonal matrix are of the form

$$
\operatorname{eig}(\tilde{R})=(1, \cos (\theta)+i \sin (\theta), \cos (\theta)-i \sin (\theta)),
$$

where $\theta$ is the angle from the angle-axis representation. Given that all the eigenvalues are real it follows that $\theta=0$ or $\theta=$ $\pm \pi$. The first possibility is the desired case $(\tilde{R}, \tilde{b})=(I, 0)$. The second possibility is the case where $\operatorname{tr}(\tilde{R})=-1$.

When $\omega_{\text {mes }} \equiv 0$ then Eqn's 32 and Eqn's 13 lead to identical error dynamics. Thus, we use the same argument as in Theorem 4.2 to prove that $\tilde{b}=0$ on the invariant set. To see that the only forward invariant subsets are the unstable equilibria as characterised in part i) of the theorem statement we introduce $\bar{R}=R \hat{R}^{T}$. Observe that

$$
\tilde{R} M=M \tilde{R}^{T} \quad \Rightarrow \quad \bar{R} M_{0}=M_{0} \bar{R}^{T}
$$

Analogous to Eq. 35, this implies $\bar{R}=I_{3}$ or $\operatorname{tr}(\bar{R})=-1$ on the set $\omega_{\text {mes }} \equiv 0$ and $\bar{R}=\bar{R}^{T}$. Set $\bar{R}^{\prime}=U_{0}^{T} \bar{R} U_{0}$. Then

$$
\bar{R}^{\prime} \Lambda-\Lambda \bar{R}^{\prime}=0 \quad \Rightarrow \quad \forall i, j\left(\lambda_{i}-\lambda_{j}\right) \bar{R}_{i j}^{\prime}=0
$$

As $M_{0}$ has three distinct eigenvalues, it follows that $\bar{R}_{i j}^{\prime}=0$ for all $i \neq j$ and thus $\bar{R}^{\prime}$ is diagonal. Therefore, there are four isolated equilibrium points $\bar{R}_{0}^{\prime}=U_{0} D_{i} U_{0}^{T}, i=1, \ldots, 3$ (where $D_{i}$ are specified in part i) of the theorem statement) and $\bar{R}^{\prime}=I$ that satisfy the condition $\omega_{\text {mes }} \equiv 0$. The case $\bar{R}_{0}^{\prime}=I=U_{0} D_{4} U_{0}^{T}$ (where $D_{4}=I$ ) corresponds to the equilibrium $(\tilde{R}, \tilde{b})=(I, 0)$ while we will show that the other three equilibria are unstable.

We proceed by computing the dynamics of the filter in the new $\bar{R}$ variable and using these dynamics to prove the stability properties of the equilibria. The dynamics associated to $\bar{R}$ are

$$
\begin{aligned}
\dot{\bar{R}} & =\dot{R} \hat{R}^{T}+R \dot{\hat{R}}^{T} \\
& =R \Omega_{\times} \hat{R}^{T}-R(\Omega+\tilde{b})_{\times} \hat{R}^{T}-k_{P} R \mathbb{P}_{a}(\tilde{R} M) \hat{R}^{T} \\
& =-R \tilde{b}_{\times} \hat{R}^{T}-\frac{k_{P}}{2} R\left(\tilde{R} M-M \tilde{R}^{T}\right) \hat{R}^{T} \\
& =-R \tilde{b}_{\times}\left(R^{T} R\right) \hat{R}^{T}-\frac{k_{P}}{2} R\left(\hat{R}^{T} M_{0} R-R^{T} M_{0} \hat{R}\right) \hat{R}^{T} \\
& =-(R \tilde{b})_{\times} \bar{R}-\frac{k_{P}}{2}\left(\bar{R} M_{0} \bar{R}-M_{0}\right)
\end{aligned}
$$


Setting $\bar{b}=R \tilde{b}$, one obtains

$$
\dot{\bar{R}}=-\bar{b}_{\times} \bar{R}-\frac{k_{P}}{2}\left(\bar{R} M_{0} \bar{R}-M_{0}\right) .
$$

The dynamics of the new estimation error on the bias $\bar{b}$ are

$$
\begin{aligned}
\dot{\bar{b}}_{\times} & =\dot{R} \bar{b}_{\times} R^{T}+R \bar{b}_{\times} \dot{R}^{T}+k_{I} R \mathbb{P}_{a}(\tilde{R} M) R^{T} \\
& =\left[(R \Omega)_{\times}, \bar{b}_{\times}\right]+\frac{k_{I}}{2} R\left(\hat{R}^{T} M_{0} R-R^{T} M_{0} \hat{R}\right) R^{T} \\
& =\left[(R \Omega)_{\times}, \bar{b}_{\times}\right]+\frac{k_{I}}{2}\left(\bar{R} M_{0}-M_{0} \bar{R}^{T}\right)
\end{aligned}
$$

The dynamics of $(\bar{R}, \bar{b})$ (Eqn's 37 and 38) are an alternative formulation of the error dynamics to $(\tilde{R}, \tilde{b})$.

Consider a first order approximation of $(\bar{R}, \bar{b})$ (Eqn's 37 and $38)$ around an equilibrium point $\left(\bar{R}_{0}, 0\right)$

$$
\bar{R}=\bar{R}_{0}\left(I_{3}+x_{\times}\right), \quad \bar{b}=-y .
$$

The linearisation of Eq. 37 is given by

$$
\bar{R}_{0} \dot{x}_{\times}=y_{\times} \bar{R}_{0}-\frac{k_{P}}{2}\left(\bar{R}_{0} x_{\times} M_{0} \bar{R}_{0}+\bar{R}_{0} M_{0} \bar{R}_{0} x_{\times}\right),
$$

and thus

$$
\dot{x}_{\times}=\bar{R}_{0}^{T} y_{\times} \bar{R}_{0}-\frac{k_{P}}{2}\left(x_{\times} M_{0} \bar{R}_{0}+M_{0} \bar{R}_{0} x_{\times}\right),
$$

and finally

$U_{0}^{T} \dot{x}_{\times} U_{0}=D_{i}\left(U_{0}^{T} y\right)_{\times} D_{i}-\frac{k_{P}}{2}\left(\left(U_{0}^{T} x\right)_{\times} \Lambda D_{i}+\Lambda D_{i}\left(U_{0}^{T} x\right)_{\times}\right)$

for $i=1, \ldots, 4$ and where $\Lambda$ is specified in part i) of the theorem statement. Define

$$
\begin{aligned}
& A_{1}=0.5 \operatorname{diag}\left(\lambda_{2}+\lambda_{3},-\lambda_{1}+\lambda_{3},-\lambda_{1}+\lambda_{2}\right) \\
& A_{2}=0.5 \operatorname{diag}\left(\lambda_{2}-\lambda_{3}, \lambda_{1}-\lambda_{3}, \lambda_{1}+\lambda_{2}\right) \\
& A_{3}=0.5 \operatorname{diag}\left(-\lambda_{2}+\lambda_{3}, \lambda_{1}+\lambda_{3},+\lambda_{1}-\lambda_{2}\right) \\
& A_{4}=0.5 \operatorname{diag}\left(-\lambda_{2}-\lambda_{3},-\lambda_{1}-\lambda_{3},-\lambda_{1}-\lambda_{2}\right)
\end{aligned}
$$

Setting $y^{\prime}=U_{0}^{T} y$ and $x^{\prime}=U_{0}^{T} x$ one may write the linearisation Eq. 37 as

$$
\dot{x}^{\prime}=k_{P} A_{i} x^{\prime}+D_{i} y^{\prime}, \quad i=1, \ldots, 4 .
$$

We continue by computing the linearisation of $\dot{\bar{b}}$. Equation (38) may be approximated to a first order by

$$
-\dot{y}_{\times}=\left[(R \Omega)_{\times},-y_{\times}\right]+\frac{k_{I}}{2}\left(\bar{R}_{0} x_{\times} M_{0}+M_{0} x_{\times} \bar{R}_{0}\right)
$$

and thus

$$
-U_{0}^{T} \dot{y}_{\times} U_{0}=\left[\left(U_{0}^{T} R \Omega\right)_{\times},-y_{\times}^{\prime}\right]+\frac{k_{I}}{2}\left(D_{i} x_{\times}^{\prime} \Lambda+\Lambda x_{\times}^{\prime} D_{i}\right) .
$$

Finally, for $i=1, \ldots, 4$

$$
U_{0}^{T} \dot{y}_{\times} U_{0}=-\frac{k_{I}}{2}\left(\left(D_{i} x^{\prime}\right)_{\times} D_{i} \Lambda+\Lambda D_{i}\left(D_{i} x^{\prime}\right)_{\times}\right)+\left[\Omega_{\times}^{\prime}, y_{\times}^{\prime}\right] \text {. }
$$

Rewriting in terms of the variables $x^{\prime}, y^{\prime}$ and setting $\Omega^{\prime}=$ $U_{0}^{T} R \Omega$ one obtains

$$
\dot{y}^{\prime}=k_{I} A_{i} D_{i} x^{\prime}+\Omega^{\prime} \times y^{\prime}, \quad \text { for } i=1, \ldots, 4 .
$$

The combined error dynamic linearisation in the primed coordinates is

$$
\left(\begin{array}{c}
\dot{x}^{\prime} \\
\dot{y}^{\prime}
\end{array}\right)=\left(\begin{array}{cc}
k_{P} A_{i} & D_{i} \\
k_{I} A_{i} D_{i} & \Omega^{\prime}(t)_{\times}
\end{array}\right)\left(\begin{array}{c}
x^{\prime} \\
y^{\prime}
\end{array}\right), \quad i=1, \ldots, 4 .
$$

To complete the proof of part i) of the theorem statement we will prove that the three equilibria associated with $\left(\bar{R}_{* i}, \bar{b}_{* i}\right)$ for $i=1,2,3$ are unstable. The demonstration is analogous to the proof of the Chetaev's Theorem (see [40, pp. 111-112]). Consider the following cost function:

$$
S=\frac{1}{2} k_{I} x^{\prime T} A_{i} x^{\prime}-\frac{1}{2}\left|y^{\prime}\right|^{2}
$$

It is straightforward to verify that its time derivative is always positive

$$
\dot{S}=k_{P} k_{I} A_{i}^{2}\left|x^{\prime}\right|^{2} .
$$

Note that for $i=1, \ldots, 3$ then $A_{i}$ has at least one element of the diagonal positive. For each $i=1, \ldots, 3$ and $r>0$, define

$$
U_{r}=\left\{\xi^{\prime}=\left(x^{\prime}, y^{\prime}\right)^{T}: S\left(\xi^{\prime}\right)>0,\left|\xi^{\prime}\right|<r\right\}
$$

and note that $U_{r}$ is non-null for all $r>0$. Let $\xi_{0}^{\prime} \in U_{r}$ such that $S\left(\xi_{0}^{\prime}\right)>0$. A trajectory $\xi^{\prime}(t)$ initialized at $\xi^{\prime}(0)=\xi_{0}^{\prime}$ will diverge from the compact set $U_{r}$ since $\dot{S}\left(\xi^{\prime}\right)>0$ on $U_{r}$. However, the trajectory cannot exit $U_{r}$ through the surface $S\left(\xi^{\prime}\right)=0$ since $S\left(\xi^{\prime}(t)\right) \geq S\left(\xi_{0}^{\prime}\right)$ along the trajectory. Restricting $r$ such that the linearisation is valid, then the trajectory must exit $U_{r}$ through the sphere $\left|\xi^{\prime}\right|=r$. Consequently, trajectories initially arbitrarily close to $(0,0)$ will diverge. This proves that the point $(0,0)$ is locally unstable.

To prove local exponential stability of $(\bar{R}, \bar{b})=(I, 0)$ we consider the linearisation Eq. 39 for $i=4$. Note that $D_{4}=I$ and $A_{4}<0$. Set $K_{P}=-\frac{k_{P}}{2} A_{4}$ and $K_{I}=-\frac{k_{I}}{2} A_{4}$. Then $K_{P}, K_{I}>0$ are positive definite and Eq. 39 may be written as

$$
\frac{d}{d t}\left(\begin{array}{c}
x^{\prime} \\
y^{\prime}
\end{array}\right)=\left(\begin{array}{cc}
-K_{P} & I_{3} \\
-K_{I} & \Omega^{\prime}(t)_{\times}
\end{array}\right)\left(\begin{array}{c}
x^{\prime} \\
y^{\prime}
\end{array}\right)
$$

Consider a cost function $V=\xi^{T} P \xi^{\prime}$ with $P$ given by Eq. 27 . Analogous to Eq. 28, the time derivative of $V$ is given by

$$
\begin{aligned}
\dot{V}= & -\left(K_{P} \alpha_{1}-\alpha_{2} K_{I}\right)\left|x^{\prime}\right|^{2}-\alpha_{2}\left|y^{\prime}\right|^{2} \\
& +y^{\prime T} x^{\prime}\left(\alpha_{1}+K_{P} \alpha_{2}-\alpha_{3} K_{I}\right)-\alpha_{2} x^{\prime T}\left(\Omega^{\prime} \times y^{\prime}\right) .
\end{aligned}
$$

Once again, it is straightforward to verify that

$$
\dot{V} \leq-2\left(\left|x^{\prime}\right|,\left|y^{\prime}\right|\right) Q\left(\begin{array}{l}
\left|x^{\prime}\right| \\
\left|y^{\prime}\right|
\end{array}\right)
$$

where $Q$ is defined in Eq. 27 and this proves local exponential stability of $(\bar{R}, \bar{b})=(I, 0)$.

The final statement of the theorem follows directly from the above results along with classical dynamical systems theory and the proof is complete.

Remark: If $n=3$, the weights $k_{i}=1$, and the measured directions are orthogonal $\left(v_{i}^{T} v_{j}=0, \forall i \neq j\right)$ then $M=I_{3}$. The cost function $E_{\text {mes }}$ becomes

$$
E_{\mathrm{mes}}=3-\operatorname{tr}(\tilde{R} M)=\operatorname{tr}\left(I_{3}-\tilde{R}\right)=E_{\mathrm{tr}} .
$$


In this case, the explicit complementary filter (Eqn's 32) and the passive complementary filter (Eqn's 13) are identical.

Remark: It is possible to weaken the assumptions in Theorem 5.1 to allow any choice of gains $k_{i}$ and any structure of the matrix $M_{0}$ and obtain analogous results. The case where all three eigenvalues of $M_{0}$ are equal is equivalent to the passive complementary filter scaled by a constant. The only other case where $n>2$ has

$$
M_{0}=U_{0} \operatorname{diag}\left(\lambda_{1}, \lambda_{1}, \lambda_{2}\right) U_{0}^{T}
$$

for $\lambda_{1}>\lambda_{2} \geq 0$. (Note that the situation where $n=1$ is considered in Corollary 5.2.) It can be shown that any symmetry $\bar{R}_{*}=\exp \left(\pi a_{* \times}\right)$ with $a_{*} \in \operatorname{span}\left\{v_{01}, v_{02}\right\}$ satisfies $\omega_{\text {mes }} \equiv 0$ and it is relatively straightforward to verify that this set is forward invariant under the closed-loop filter dynamics. This invalidates part i) of Theorem 5.1 as stated, however, it can be shown that the new forward invariant points are unstable as expected. To see this, note that any $\left(\bar{R}_{*}, \bar{b}_{*}\right)$ in this set corresponds to the minimal cost of $E_{\text {mes }}$ on $\mathbb{U}_{0}$. Consequently, any neighbourhood of $\left(\bar{R}_{*}, \bar{b}_{*}\right)$ contains points $(\tilde{R}, \tilde{b})$ such that $V(\tilde{R}, \tilde{b})<V\left(\bar{R}_{*}, \bar{b}_{*}\right)$ and the Lyapunov decrease condition ensures instability. There is still a separate isolated unstable equilibrium in $\mathbb{U}_{0}$, and the stable equilibrium, that must be treated in the same manner as undertaken in the formal proof of Theorem 5.1. Following through the proof yields analogous results to Theorem 5.1 for arbitrary choice of gains $\left\{k_{i}\right\}$.

The two typical measurements obtained from an IMU unit are estimates of the gravitational, $a$, and magnetic, $m$, vector fields

$$
v_{a}=R^{T} \frac{a_{0}}{\left|a_{0}\right|}, \quad v_{m}=R^{T} \frac{m_{0}}{\left|m_{0}\right|} .
$$

In this case, the cost function $E_{\text {mes }}$ becomes

$$
E_{\text {mes }}=k_{1}\left(1-\left\langle\hat{v}_{a}, v_{a}\right\rangle\right)+k_{2}\left(1-\left\langle\hat{v}_{m}, v_{m}\right\rangle\right)
$$

The weights $k_{1}$ and $k_{2}$ are introduced to weight the confidence in each measure. In situations where the IMU is subject to high magnitude accelerations (such as during takeoff or landing manoeuvres) it may be wise to reduce the relative weighting of the accelerometer data $\left(k_{1}<<k_{2}\right)$ compared to the magnetometer data. Conversely, in many applications the IMU is mounted in the proximity to powerful electric motors and their power supply busses leading to low confidence in the magnetometer readings (choose $k_{1}>>k_{2}$ ). This is a very common situation in the case of mini aerial vehicles with electric motors. In extreme cases the magnetometer data is unusable and provides motivation for a filter based solely on accelerometer data.

\section{A. Estimation from the measurements of a single direction}

Let $v_{a}$ be a measured body fixed frame direction associated with a single inertial direction $v_{0 a}, v_{a}=R^{T} v_{0 a}$. Let $\hat{v}_{a}$ be an estimate $\hat{v}_{a}=\hat{R}^{T} v_{0 a}$. The error considered is

$$
E_{\text {mes }}=1-\operatorname{tr}(\tilde{R} M) ; \quad M=R^{T} v_{0 a} v_{0 a}^{T} R
$$

Corollary 5.2: Consider the rotation kinematics Eq. 4 for a time-varying $R(t) \in S O(3)$ and with measurements given by
Eqn's 29 (for a single measurement $v_{1}=v_{a}$ ) and Eq. 11b. Let $(\hat{R}(t), \hat{b}(t))$ denote the solution of Eqn's 32 . Assume that $\Omega(t)$ is a bounded, absolutely continuous signal and $\left(\Omega(t), v_{a}(t)\right)$ are asymptotically independent (see $\S$ II-A). Define

$$
\mathbb{U}_{1}=\left\{(\tilde{R}, \tilde{b}): v_{0 a}^{T} \tilde{R} v_{0 a}=-1, \tilde{b}=0\right\} .
$$

Then:

1) The set $\mathbb{U}_{1}$ is forward invariant and unstable under the closed-loop filter dynamics.

2) The estimate $\left(\hat{v}_{a}, \hat{b}\right)$ is locally exponentially stable to $\left(v_{a}, b\right)$.

3) For almost all initial conditions $\left(\tilde{R}_{0}, \tilde{b}_{0}\right) \notin \mathbb{U}_{1}$ then $\left(\hat{v}_{a}, \hat{b}\right)$ converges to the trajectory $\left(v_{a}(t), b\right)$.

Proof: The dynamics of $\hat{v}_{a}$ are given by

$$
\dot{\hat{v}}_{a}=-\left(\Omega+\tilde{b}+k_{P} v_{a} \times \hat{v}_{a}\right) \times \hat{v}_{a}
$$

Define the following storage function

$$
V=E_{\mathrm{mes}}+\frac{1}{k_{I}} \tilde{b}^{2} .
$$

The derivative of $V$ is given by

$$
\dot{V}=-k_{P}\left\|\left(v_{a} \times \hat{R}^{T} v_{0 a}\right)_{\times}\right\|^{2}=-2 k_{P}\left|v_{a} \times \hat{v}_{a}\right|^{2}
$$

The Lyapunov-like function $V$ derivative is negative semidefinite ensuring that $\tilde{b}$ is bounded and $v_{a} \times \hat{v}_{a} \rightarrow 0$. The set $v_{a} \times \hat{v}_{* a}=0$ is characterised by $v_{a}= \pm \hat{v}_{* a}$ and thus

$$
\hat{v}_{* a}^{T} v_{a}= \pm 1=v_{0 a}^{T} \hat{R}_{*}^{T} R v_{0 a}=v_{0 a}^{T} \tilde{R}_{*} v_{0 a} .
$$

Consider a trajectory $\left(\hat{v}_{* a}(t), b_{*}(t)\right)$ that satisfies the filter dynamics and for which $\hat{v}_{* a}= \pm v_{a}$ for all time. One has

$$
\begin{aligned}
\frac{d}{d t}\left(v_{a} \times \hat{v}_{* a}\right) & =0 \\
& =-\left(\Omega \times v_{a}\right) \times \hat{v}_{* a}-v_{a} \times\left(\Omega \times \hat{v}_{* a}\right) \\
& -v_{a} \times\left(\tilde{b}_{*} \times \hat{v}_{* a}\right)-k_{P} v_{a} \times\left(\left(v_{a} \times \hat{v}_{* a}\right) \times \hat{v}_{* a}\right) \\
& = \pm v_{a} \times\left(\tilde{b}_{*} \times v_{a}\right)=0 .
\end{aligned}
$$

Differentiating this expression again one obtains

$$
\left(\left(\Omega \times v_{a}\right) \times\left(\tilde{b}_{*} \times v_{a}\right)+v_{a} \times\left(\tilde{b}_{*} \times\left(\Omega \times v_{a}\right)\right)\right)=0
$$

Since the signals $\Omega$ and $v_{a}$ are asymptotically independent it follows that the functional expression on the left hand side is degenerate. This can only hold if $\tilde{b}_{*} \equiv 0$. For $\hat{v}_{* a}=-v_{a}$, this set of trajectories is characterised by the definition of $\mathbb{U}_{1}$. It is straightforward to adapt the arguments in Theorems 4.1 and 4.2 to see that this set is forward invariant. Note that for $\tilde{b}_{*}=0$ then $V=E_{\text {mes }}$. It is direct to see that $\left(\hat{v}_{* a}(t), b_{*}(t)\right)$ lies on a local maximum of $E_{\mathrm{mes}}$ and that any neighbourhood contains points such that the full Lyapunov function $V$ is strictly less than its value on the set $\mathbb{U}_{1}$. This proves instability of $\mathbb{U}_{1}$ and completes part i) of the corollary.

The proof of part ii) and part iii) is analogous to the proof of Theorem 5.1 (see also [15]).

An important aspect of Corollary 5.2 is the convergence of the bias terms in all degrees of freedom. This ensures that, for a real world system, the drift in the attitude estimate around the unmeasured axis $v_{0 a}$ will be driven asymptotically by a zero mean noise process rather than a constant bias term. This makes the proposed filter a practical algorithm for a wide range of MAV applications. 


\section{EXPERIMENTAL RESULTS}

In this section, we present experimental results to demonstrate the performance of the proposed observers.

Experiments were undertaken on two real platforms to demonstrate the convergence of the attitude and gyro bias estimates.

1) The first experiment was undertaken on a robotic manipulator with an IMU mounted on the end effector and supplied with synthetic estimates of the magnetic field measurement. The robotic manipulator was programmed to simulate the movement of a flying vehicle in hovering flight regime. The filter estimates are compared to orientation measurements computed from the forward kinematics of the manipulator. Only the passive and direct complimentary filters were run on this test bed.

2) The second experiment was undertaken on the VTOL MAV HoverEye ${ }^{\circledR}$ developed by Bertin Technologies (Figure 1). The VTOL belongs to the class of 'sit on tail' ducted fan VTOL MAV, like the iSTAR9 and Kestrel developed respectively by Allied Aerospace [41] and Honeywell [42]. It was equipped with a low-cost IMU that consists of 3-axis accelerometers and 3-axis gyroscopes. Magnetometers were not integrated in the MAV due to perturbations caused by electrical motors. The explicit complementary filter was used in this experiment.

For both experiments the gains of the proposed filters were chosen to be: $k_{P}=1 \mathrm{rad} . \mathrm{s}^{-1}$ and $k_{I}=0.3 \mathrm{rad} . \mathrm{s}^{-1}$. The inertial data was acquired at rates of $25 \mathrm{~Hz}$ for the first experiment and $50 \mathrm{~Hz}$ for the second experiment. The quaternion version of the filters (Appendix B) were implemented with first order Euler numerical integration followed by rescaling to preserve the unit norm condition.

Experimental results for the direct and passive versions of the filter are shown in Figures 6 and 7. In Figure 6 the only significant difference between the two responses lies in the initial transient responses. This is to be expected, since both filters will have the same theoretical asymptotic performance. In practice, however, the increased sensitivity of the direct filter to noise introduced in the computation of the measured rotation $R_{y}$ is expected to contribute to slightly higher noise in this filter compared to the passive.

The response of the bias estimates is shown in Figure 7. Once again the asymptotic performance of the filters is similar after an initial transient. From this figure it is clear that the passive filter displays slightly less noise in the bias estimates than for the direct filter (note the different scales in the $y$-axis).

Figures 8 and 9 relate to the second experiment. The experimental flight of the MAV was undertaken under remote control by an operator. The experimental flight plan used was: First, the vehicle was located on the ground, initially headed toward $\psi(0)=0$. After take off, the vehicle was stabilized in hovering condition, around a fixed heading which remains close the initial heading of the vehicle on the ground. Then, the operator engages $\mathrm{a} \simeq 90^{\circ}$-left turn manoeuvre, returns to the initial heading, and follows with $\mathrm{a} \simeq 90^{\circ}$-right turn
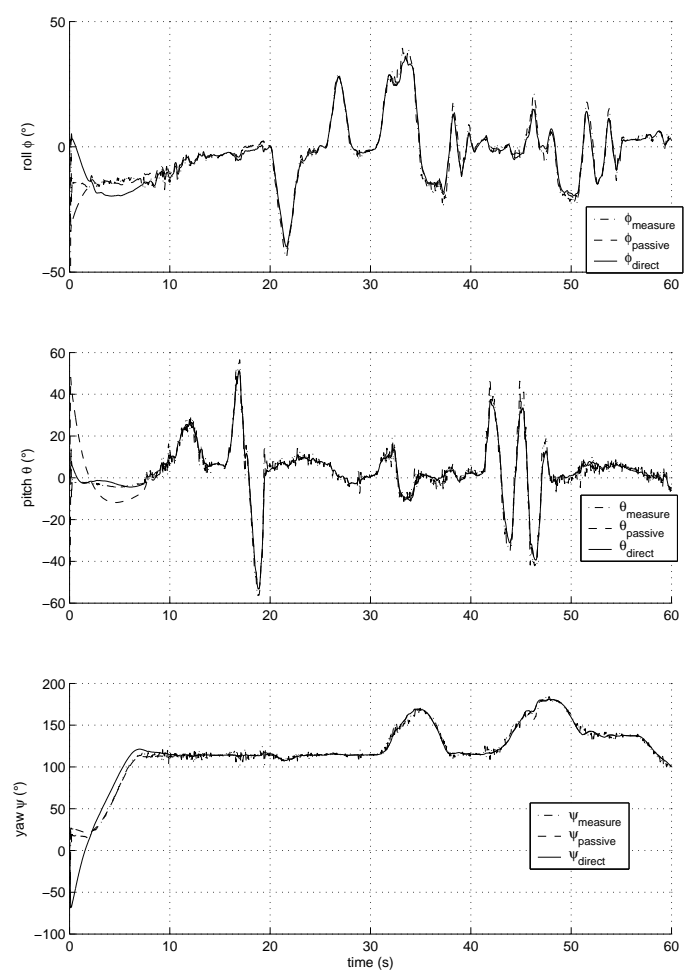

Fig. 6. Euler angles from direct and passive complementary filters
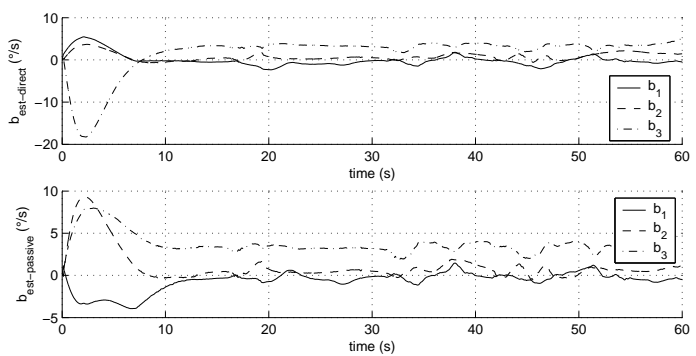

Fig. 7. Bias estimation from direct and passive complementary filters

manoeuvre, before returning to the initial heading and landing the vehicle. After landing, the vehicle is placed by hand at its initial pose such that final and initial attitudes are the identical.

Figure 8 plots the pitch and roll angles $(\phi, \theta)$ estimated directly from the accelerometer measurements against the estimated values from the explicit complementary filter. Note the large amounts of high frequency noise in the raw attitude estimates. The plots demonstrate that the filter is highly successful in reconstructing the pitch and roll estimates.

Figure 9 presents the gyros bias estimation verses the predicted yaw angle $(\phi)$ based on open loop integration of the gyroscopes. Note that the explicit complementary filter here is based solely on estimation of the gravitational direction. Consequently, the yaw angle is the indeterminate angle that is not directly stabilised in Corollary 5.2. Figure 9 demonstrates that the proposed filter has successfully identified the bias of the yaw axis gyro. The final error in yaw orientation of the microdrone after landing is less than 5 degrees over a two minute flight. Much of this error would be due to the initial transient when the bias estimate was converging. Note that the 
second part of the figure indicates that the bias estimates are not constant. Although some of this effect may be numerical, it is also to be expected that the gyro bias on low cost IMU systems are highly susceptible to vibration effects and changes in temperature. Under flight conditions changing engine speeds and aerodynamic conditions can cause quite fast changes in gyro bias.
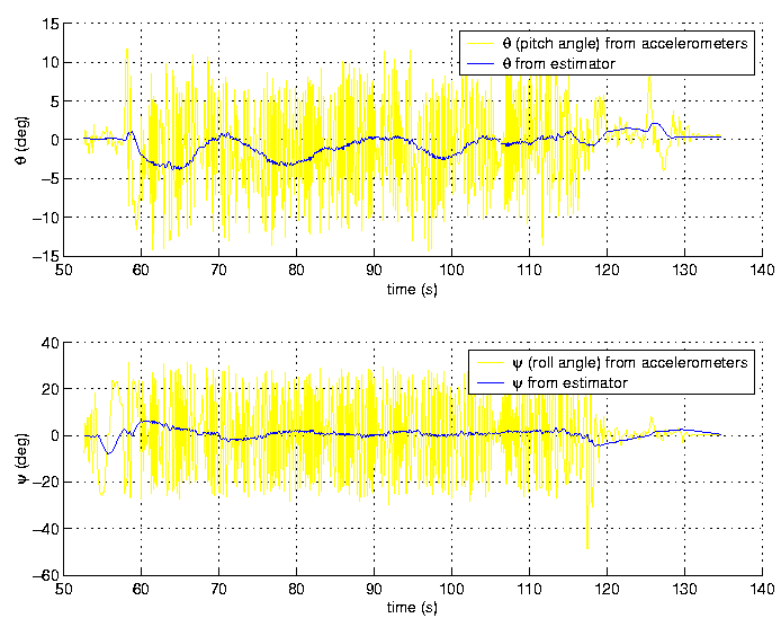

Fig. 8. Estimation results of the Pitch and roll angles.
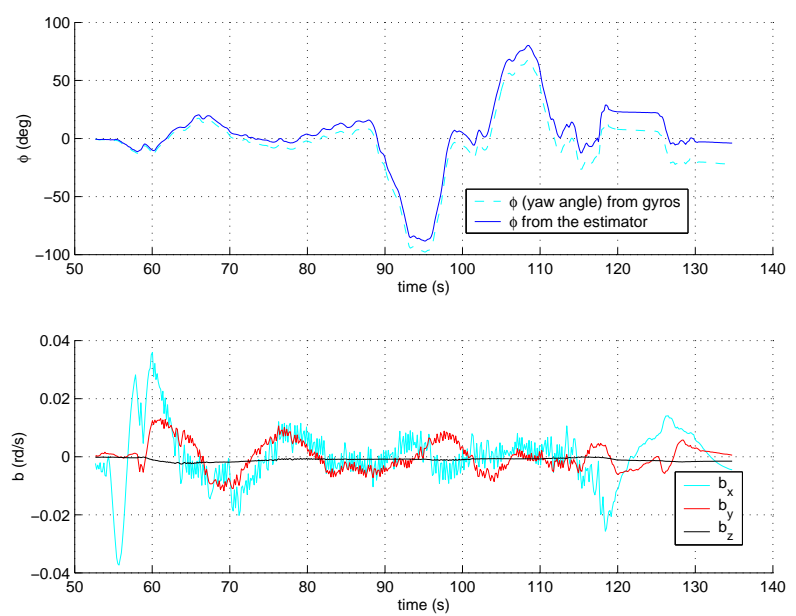

Fig. 9. Gyros bias estimation and influence of the observer on yaw angle.

\section{CONCLUSION}

This paper presents a general analysis of attitude observer design posed directly on the special orthogonal group. Three non-linear observers, ensuring almost global stability of the observer error, are proposed:

Direct complementary filter: A non-linear observer posed on $S O(3)$ that is related to previously published non-linear observers derived using the quaternion representation of $S O(3)$.

Passive complementary filter: A non-linear filter equation that takes advantage of the symmetry of $S O(3)$ to avoid transformation of the predictive angular velocity term into the estimator frame of reference. The resulting observer kinematics are considerably simplified and avoid coupling of constructed attitude error into the predictive velocity update.

Explicit complementary filter: A reformulation of the passive complementary filter in terms of direct vectorial measurements, such as gravitational or magnetic field directions obtained for an IMU. This observer does not require online algebraic reconstruction of attitude and is ideally suited for implementation on embedded hardware platforms. Moreover, the filter remains well conditioned in the case where only a single vector direction is measured.

The performance of the observers was demonstrated in a suite of experiments. The explicit complementary filter is now implemented as the primary attitude estimation system on several MAV vehicles world wide.

\section{APPENDIX A}

\section{A REVIEW OF COMPLEMENTARY FILTERING}

Complementary filters provide a means to fuse multiple independent noisy measurements of the same signal that have complementary spectral characteristics [11]. For example, consider two measurements $y_{1}=x+\mu_{1}$ and $y_{2}=x+\mu_{2}$ of a signal $x$ where $\mu_{1}$ is predominantly high frequency noise and $\mu_{2}$ is a predominantly low frequency disturbance. Choosing a pair of complementary transfer functions $F_{1}(s)+F_{2}(s)=1$ with $F_{1}(s)$ low pass and $F_{2}(s)$ high pass, the filtered estimate is given by

$\hat{X}(s)=F_{1}(s) Y_{1}+F_{2}(s) Y_{2}=X(s)+F_{1}(s) \mu_{1}(s)+F_{2}(s) \mu_{2}(s)$.

The signal $X(s)$ is all pass in the filter output while noise components are high and low pass filtered as desired. This type of filter is also known as distorsionless filtering since the signal $x(t)$ is not distorted by the filter [43]. Complementary filters are particularly well suited to fusing low bandwidth position measurements with high band width rate measurements for first order kinematic systems. Consider the linear kinematics

$$
\dot{x}=u \text {. }
$$

with typical measurement characteristics

$$
y_{x}=L(s) x+\mu_{x}, \quad y_{u}=u+\mu_{u}+b(t)
$$

where $L(s)$ is low pass filter associated with sensor characteristics, $\mu$ represents noise in both measurements and $b(t)$ is a deterministic perturbation that is dominated by low-frequency content. Normally the low pass filter $L(s) \approx 1$ over the frequency range on which the measurement $y_{x}$ is of interest. The rate measurement is integrated $\frac{y_{u}}{s}$ to obtain an estimate of the state and the noise and bias characteristics of the integrated signal are dominantly low frequency effects. Choosing

$$
\begin{aligned}
& F_{1}(s)=\frac{C(s)}{C(s)+s} \\
& F_{2}(s)=1-F_{1}(s)=\frac{s}{C(s)+s}
\end{aligned}
$$


with $C(s)$ all pass such that $L(s) F_{1}(s) \approx 1$ over the bandwidth of $L(s)$. Then

$$
\hat{X}(s) \approx X(s)+F_{1}(s) \mu_{x}(s)+\frac{\mu_{u}(s)+b(s)}{C(s)+s}
$$

Note that even though $F_{2}(s)$ is high pass the noise $\mu_{u}(s)+b(s)$ is low pass filtered. In practice, the filter structure is implemented by exploiting the complementary sensitivity structure of a linear feedback system subject to load disturbance. Consider the block diagram in Figure 10. The output $\hat{x}$ can

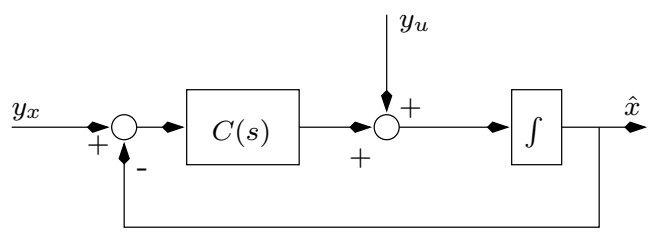

Fig. 10. Block diagram of a classical complementary filter.

be written

$$
\begin{aligned}
\hat{x}(s) & =\frac{C(s)}{s+C(s)} y_{x}(s)+\frac{s}{C(s)+s} \frac{y_{u}(s)}{s} \\
& =T(s) y_{x}(s)+S(s) \frac{y_{u}(s)}{s}
\end{aligned}
$$

where $S(s)$ is the sensitivity function of the closed-loop system and $T(s)$ is the complementary sensitivity. This architecture is easy to implement efficiently and allows one to use classical control design techniques for $C(s)$ in the filter design. The simplest choice is a proportional feedback $C(s)=k_{P}$. In this case the closed-loop dynamics of the filter are given by

$$
\dot{\hat{x}}=y_{u}+k_{P}\left(y_{x}-\hat{x}\right) \text {. }
$$

The frequency domain complementary filters associated with this choice are $F_{1}(s)=\frac{k_{P}}{s+k_{P}}$ and $F_{2}(s)=\frac{s}{s+k_{P}}$. Note that the crossover frequency for the filter is at $k_{P}$ rad.s $^{-1}$. The gain $k_{P}$ is typically chosen based on the low pass characteristics of $y_{x}$ and the low frequency noise characteristics of $y_{u}$ to choose the best crossover frequency to tradeoff between the two measurements. If the rate measurement bias, $b(t)=b_{0}$, is a constant then it is natural to add an integrator to the compensator to make the system type I

$$
C(s)=k_{P}+\frac{k_{I}}{s} \text {. }
$$

A type I system will reject the constant load disturbance $b_{0}$ from the output. Gain design for $k_{P}$ and $k_{I}$ is typically based on classical frequency design methods. The non-linear development in the body of the paper requires a Lyapunov analysis of closed-loop system Eq. 43. Applying the PI compensator, Eq. 44, one obtains state space filter with dynamics

$$
\dot{\hat{x}}=y_{u}-\hat{b}+k\left(y_{x}-\hat{x}\right), \quad \dot{\hat{b}}=-k_{I}\left(y_{x}-\hat{x}\right)
$$

The negative sign in the integrator state is introduced to indicate that the state $\hat{b}$ will cancel the bias in $y_{u}$. Consider the Lyapunov function

$$
\mathcal{L}=\frac{1}{2}|x-\hat{x}|^{2}+\frac{1}{2 k_{I}}\left|b_{0}-\hat{b}\right|^{2}
$$

Abusing notation for the noise processes, and using $\tilde{x}=(x-$ $\hat{x})$, and $\tilde{b}=\left(b_{0}-\hat{b}\right)$, one has

$$
\frac{d}{d t} \mathcal{L}=-k_{P}|\tilde{x}|^{2}-\mu_{u} \tilde{x}+\mu_{x}(\tilde{b}-k \tilde{x})
$$

In the absence of noise one may apply Lyapunov's direct method to prove convergence of the state estimate. LaSalle's principal of invariance may be used to show that $\hat{b} \rightarrow b_{0}$. When the underlying system is linear, then the linear form of the feedback and adaptation law ensure that the closed-loop system is linear and stability implies exponential stability.

\section{APPENDIX B \\ QUATERNION REPRESENTATIONS OF OBSERVERS}

The unit quaternion representation of rotations is commonly used for the realisation of algorithms on $S O(3)$ since it offers considerable efficiency in code implementation. The set of quaternions is denoted $\mathbb{Q}=\left\{q=(s, v) \in \mathbb{R} \times \mathbb{R}^{3}:|q|=1\right\}$. The set $\mathbb{Q}$ is a group under the operation

$$
q_{1} \otimes q_{2}=\left[\begin{array}{c}
s_{1} s_{2}-v_{1}^{T} v_{2} \\
s_{1} v_{2}+s_{2} v_{1}+v_{1} \times v_{2}
\end{array}\right]
$$

with identity element $\mathbf{1}=(1,0,0,0)$. The group of quaternions are homomorphic to $S O(3)$ via the map

$$
F: \mathbb{Q} \rightarrow S O(3), \quad F(q):=I_{3}+2 s v_{\times}+2 v_{\times}^{2}
$$

This map is a two to one mapping of $\mathbb{Q}$ onto $S O(3)$ with kernel $\{(1,0,0,0),(-1,0,0,0)\}$, thus, $\mathbb{Q}$ is locally isomorphic to $S O(3)$ via $F$. Given $R \in S O(3)$ such that $R=\exp \left(\theta a_{\times}\right)$ then $F^{-1}(R)=\left\{ \pm\left(\cos \left(\frac{\theta}{2}\right), \sin \left(\frac{\theta}{2}\right) a\right)\right\}$ Let $\Omega \in\{A\}$ denote a body-fixed frame velocity, then the pure quaternion $\mathbf{p}(\Omega)=(0, \Omega)$ is associated with a quaternion velocity. Consider the rotation kinematics on $S O(3)$ Eq. 4, then the associated quaternion kinematics are given by

$$
\dot{q}=\frac{1}{2} q \otimes \mathbf{p}(\Omega)
$$

Let $q_{y} \approx q$ be a low frequency measure of $q$, and $\Omega_{y} \approx \Omega+b$ (for constant bias $b$ ) be the angular velocity measure. Let $\hat{q}$ denote the observer estimate and quaternion error $\tilde{q}$

$$
\tilde{q}=\hat{q}^{-1} \otimes q=\left[\begin{array}{c}
\tilde{s} \\
\tilde{v}
\end{array}\right]
$$

Note that

$$
2 \tilde{s} \tilde{v}=2 \cos (\theta / 2) \sin (\theta / 2) a=\frac{1}{2}(\sin \theta) a=\operatorname{vex}\left(\mathbb{P}_{a}(\tilde{R})\right)
$$

where $(\theta, a)$ is the angle axis representation of $\tilde{R}=F(\tilde{q})$. The quaternion representations of the observers proposed in this paper are:

Direct complementary filter (Eq. 12):

$$
\begin{aligned}
& \dot{\hat{q}}=\frac{1}{2} \hat{q} \otimes \mathbf{p}\left(\tilde{R}\left(\Omega_{y}-\hat{b}\right)+2 k_{P} \tilde{s} \tilde{v}\right) \\
& \dot{\hat{b}}=-2 k_{I} \tilde{s} \tilde{v}
\end{aligned}
$$


Passive complementary filter (Eq. 13):

$$
\begin{aligned}
& \dot{\hat{q}}=\frac{1}{2} \hat{q} \otimes \mathbf{p}\left(\Omega_{y}-\hat{b}+2 k_{P} \tilde{s} \tilde{v}\right) \\
& \dot{\hat{b}}=-2 k_{I} \tilde{s} \tilde{v}
\end{aligned}
$$

Explicit complementary filter (Eq. 32):

$$
\begin{aligned}
\omega_{\text {mes }} & =-\operatorname{vex}\left(\sum_{i=1}^{n} \frac{k_{i}}{2}\left(v_{i} \hat{v}_{i}^{T}-\hat{v}_{i} v_{i}^{T}\right)\right) \\
\dot{\hat{q}} & =\frac{1}{2} \hat{q} \otimes \mathbf{p}\left(\Omega_{y}-\hat{b}+k_{P} \omega_{\text {mes }}\right) \\
\dot{\hat{b}} & =-k_{I} \omega_{\text {mes }}
\end{aligned}
$$

The error dynamics associated with the direct filter expressed in the quaternion formulation are

$$
\dot{\tilde{q}}=-\frac{1}{2}\left(\mathbf{p}\left(\tilde{b}+k_{P} \tilde{s} \tilde{v}\right) \otimes \tilde{q}\right) .
$$

The error dynamics associated with the passive filter are

$$
\dot{\tilde{q}}=\frac{1}{2}\left(\tilde{q} \otimes \mathbf{p}(\Omega)-\mathbf{p}(\Omega) \otimes \tilde{q}-\mathbf{p}\left(\tilde{b}+k_{P} \tilde{s} \tilde{v}\right) \otimes \tilde{q}\right) .
$$

There is a fifteen year history of using the quaternion representation and Lyapunov design methodology for filtering on $S O(3)$ (for example cf. [9], [30], [32]). To the authors knowledge the Lyapunov analysis in all cases has been based around the cost function

$$
\Phi(\tilde{q})=(|\tilde{s}|-1)^{2}+|\tilde{v}|^{2} .
$$

Due to the unit norm condition it is straightforward to show that

$$
\Phi(\tilde{q})=2(1-|\tilde{s}|)=2(1-|\cos (\theta / 2)|)
$$

The cost function proposed in this paper is $E_{\mathrm{tr}}=(1-$ $\cos (\theta))$ (Eq. 3). It is straightforward to see that the quadratic approximation of both cost functions around the point $\theta=0$ is the quadratic $\theta^{2} / 2$. The quaternion cost function $\Phi$, however, is non-differentiable at the point $\theta= \pm \pi$ while the cost $\operatorname{tr}(I-\tilde{R})$ has a smooth local maxima at this point. To the authors understanding, all quaternion filters in the published literature have a similar flavour that dates back to the seminal work of Salcudean [32]. The closest published work to that undertaken in the present paper was published by Thienel in her doctoral dissertation [44] and transactions paper [30]. The filter considered by Thienel $e t a l$. is given by

$$
\begin{aligned}
& \dot{\hat{q}}=\frac{1}{2} \hat{q} \otimes \mathbf{p}\left(\tilde{R}\left(\Omega_{y}-\hat{b}+k_{P} \operatorname{sgn}(\tilde{s}) \tilde{v}\right)\right) \\
& \dot{\hat{b}}=-k_{I} \operatorname{sgn}(\tilde{s}) \tilde{v}
\end{aligned}
$$

The $\operatorname{sgn}(\tilde{s})$ term enters naturally in the filter design from the differential, $\frac{d}{d t}|\tilde{s}|=\operatorname{sgn}(\tilde{s}) \frac{d}{d t} \tilde{s}$, of the absolute value term in the cost function $\Phi$, during the Lyapunov design process. Consider the observer obtained by replacing $\operatorname{sgn}(\tilde{s})$ in Eqn's 51 by $2 \tilde{s}$. Note that with this substitution, Eq. $51 \mathrm{~b}$ is transformed into Eq. 46b. To show that Eq. 51a transforms to Eq. 46a it is sufficient to show that $\tilde{R} \tilde{v}=\tilde{v}$. This is straightforward from

$$
\begin{aligned}
2 \tilde{s} \tilde{R} \tilde{v} & =\tilde{R}(2 \tilde{s} \tilde{v})=\tilde{R} \operatorname{vex}\left(\mathbb{P}_{a}(\tilde{R})\right) \\
& =\operatorname{vex}\left(\tilde{R} \mathbb{P}_{a}(\tilde{R}) \tilde{R}^{T}\right)=\operatorname{vex}\left(\mathbb{P}_{a}(\tilde{R})\right)=2 \tilde{s} \tilde{v}
\end{aligned}
$$

This demonstrates that the quaternion filter Eqn's 51 is obtained from the standard form of the complimentary filter proposed Eq. 12 with the correction term Eq. 12c replaced by

$$
\omega_{q}=\operatorname{sgn}(\tilde{s}) \tilde{v}, \quad \tilde{q} \in F^{-1}\left(\hat{R}^{T} R\right) .
$$

Note that the correction term defined in Eq. 12c can be written $\omega=2 \tilde{s} \tilde{v}$. It follows that

$$
\omega_{q}=\frac{\operatorname{sgn}(\tilde{s})}{2 \tilde{s}} \omega
$$

The correction term for the two filters varies only by the positive scaling factor $\operatorname{sgn}(\tilde{s}) /(2 \tilde{s})$. The quaternion correction term $\omega_{q}$ is not well defined for $\tilde{s}=0$ (where $\theta= \pm \pi$ ) and these points are not well defined in the filter dynamics Eq. 51. It should be noted, however, that $\left|\omega_{q}\right|$ is bounded at $\tilde{s}=0$ and, apart from possible switching behaviour, the filter can still be implemented on the remainder of $S O(3) \times \mathbb{R}^{3}$. An argument for the use of the correction term $\omega_{q}$ is that the resulting error dynamics strongly force the estimate away from the unstable set $\mathbb{U}$ (cf. Eq. 14). An argument against its use is that, in practice, such situations will only occur due to extreme transients that would overwhelm the bounded correction term $\omega_{q}$ in any case, and cause the numerical implementation of the filter to deal with a discontinuous argument. In practice, it is an issue of little significance since the filter will general work sufficiently well to avoid any issues with the unstable set $\mathbb{U}$. For $\tilde{s} \rightarrow 1$, corresponding to $\theta=0$, the correction term $\omega_{q}$ scales to a factor of $1 / 2$ the correction term $\omega$. A simple scaling factor like this is compensated for the in choice of filter gains $k_{P}$ and $k_{I}$ and makes no difference to the performance of the filter.

\section{REFERENCES}

[1] J. Vaganay, M. Aldon, and A. Fournier, "Mobile robot attitude estimation by fusion of inertial data," in Proceedigns of the IEEE Internation Conference on Robotics and Automation ICRA, vol. 1, 1993, pp. 277282.

[2] E. Foxlin, M. Harrington, and Y. Altshuler, "Miniature 6-DOF inertial system for tracking HMD," in Proceedings of the SPIE, vol. 3362, Orlando, Florida, 1998, pp. 214-228.

[3] J. Balaram, "Kinematic observers for articulated robers," in Proceddings of the IEEE International Conference on Robotics and Automation, 2000, pp. 2597-2604.

[4] J. L. Marins, X. Yun, E. R. Backmann, R. B. McGhee, and M. Zyda, "An extended kalman filter for quaternion-based orientation estimation using marg sensors," in IEEE/RSJ International Conference on Intelligent Robots and Systems, 2001, pp. 2003-2011.

[5] E. Lefferts, F. Markley, and M. Shuster, "Kalman filtering for spacecraft attitude estimation," AIAA Journal of Guidance, Control and Navigation, vol. 5, no. 5, pp. 417-429, September 1982.

[6] B. Barshan and H. Durrant-Whyte, "Inertial navigation systems for mobile robots," IEEE Transactions on Robotics and Automation, vol. 44, no. 4, pp. 751-760, 1995.

[7] M. Zimmerman and W. Sulzer, "High bandwidth orientation measurement and control ased on complementary filtering," in Proceedings of Symposium on Roboitcs and Control, SYROCO, Vienna, Austria, 1991.

[8] A.-J. Baerveldt and R. Klang, "A low-cost and low-weight attitude estimation system for an autonomous helicopter," Intelligent Engineering Systems, 1997.

[9] B. Vik and T. Fossen, "A nonlinear observer for gps and ins integration," in Proceedings of teh IEEE Conference on Decisioin and Control, Orlando, Florida, USA, December 2001, pp. 2956-2961.

[10] H. Rehbinder and X. Hu, "Nonlinear state estimation for rigid body motion with low-pass sensors," Systems and Control Letters, vol. 40, no. 3, pp. 183-190, 2000. 
[11] E. R. Bachmann, J. L. Marins, M. J. Zyda, R. B. Mcghee, and X. Yun "An extended kalman filter for quaternion-based orientation estimation using MARG sensors," 2001.

[12] J.-M. Pflimlin, T. Hamel, P. Soueeres, and N. Metni, "Nonlinear attitude and gyroscoples bias estimation for a VTOL UAV," in Proceedings of the IFAC world congress, 2005.

[13] H. Rehbinder and X. Hu, "Drift-free attitude estimation for accelerated rigid bodies," Automatica, 2004.

[14] N. Metni, J.-M. Pflimlin, T. Hamel, and P. Soueeres, "Attitude and gyro bias estimation for a flying UAV," in IEEE/RSJ International Conference on Intelligent Robots and Systems, August 2005, pp. 295-301.

[15] — "Attitude and gyro bias estimation for a VTOL UAV," in Control Engineering Practice, 2006, p. to appear.

[16] J. Lobo and J. Dias, "Vision and inertial sensor cooperation using gravity as a vertical reference," IEEE Transactions on Pattern Analysis and Machine Intelligence, vol. 25, no. 12, pp. 1597-1608, Dec. 2003.

[17] H. Rehbinder and B. Ghosh, "Pose estimation using line-based dynamic vision and inertial sensors," IEEE Transactions on Automatic Control, vol. 48, no. 2, pp. 186-199, Feb. 2003.

[18] J.-H. Kim and S. Sukkarieh, "Airborne simultaneous localisation and map building," in Proceedings of the IEEE International Conference on Robotics and Automation, Taipei, Taiwan, September 2003, pp. 406-411.

[19] P. Corke, J. Dias, M. Vincze, and J. Lobo, "Integration of vision and inertial sensors," in Proceedings of the IEEE International Conference on Robotics and Automation, ICRA '04., ser. W-M04, Barcellona, Spain, April 2004, full day Workshop.

[20] R. Phillips and G. Schmidt, System Implications and Innovative Applications of Satellite Navigation, ser. AGARD Lecture Series 207. helpesti.nasa.gov: NASA Center for Aerospace Information, 1996, vol. 207, ch. GPS/INS Integration, pp. 0.1-0.18.

[21] D. Gebre-Egziabher, R. Hayward, and J. Powell, "Design of multi-sensor attitude determination systems," IEEE Transactions on Aerospace and Electronic Systems, vol. 40, no. 2, pp. 627-649, April 2004.

[22] J. L. Crassidis, F. L. Markley, and Y. Cheng, "Nonlinear attitude filtering methods," Journal of Guidance, Control,and Dynamics, vol. 30, no. 1, pp. 12-28, January 2007.

[23] M. Jun, S. Roumeliotis, and G. Sukhatme, "State estimation of an autonomous helicopter using Kalman filtering," in Proc. 1999 IEEE/RSJ International Conference on Robots and Systems (IROS 99), 1999.

[24] G. S. Sukhatme and S. I. Roumeliotis, "State estimation via sensor modeling for helicopter control using an indirect kalman filter," 1999.

[25] G. S. Sukhatme, G. Buskey, J. M. Roberts, P. I. Corke, and S. Saripalli, "A tale of two helicopters," in IEEE/RSJ, International Robots and Systems, Los Vegas, USA, Oct. 2003, pp. 805-810, http://wwwrobotics.usc.edu/ srik/papers/iros2003.pdf.

[26] J. M. Roberts, P. I. Corke, and G. Buskey, "Low-cost flight control system for small autonomous helicopter," in Australian Conference on Robotics and Automation, Auckland, 27-29 Novembre, 2002, pp. 71-76.

[27] P. Corke, "An inertial and visual sensing system for a small autonomous helicopter," J. Robotic Systems, vol. 21, no. 2, pp. 43-51, February 2004.

[28] G. Creamer, "Spacecraft attitude determination using gyros and quaternion measurements," The Journal of Astronautical Sciences, vol. 44, no. 3, pp. 357-371, July 1996.

[29] D. Bayard, "Fast observers for spacecraft pointing control," in Proceedings of the IEEE Conference on Decision and Control, Tampa, Florida, USA, 1998, pp. 4702-4707.

[30] J. Thienel and R. M. Sanner, "A coupled nonlinear spacecraft attitude controller and observer with an unknow constant gyro bias and gyro noise," IEEE Transactions on Automatic Control, vol. 48, no. 11, pp. 2011 - 2015, Nov. 2003

[31] G.-F. Ma and X.-Y. Jiang, "Spacecraft attitude estimation from vector measurements using particle filter," in Proceedings of the fourth Internation conference on Machine Learning and Cybernetics, Guangzhou, China, August 2005, pp. 682-687.

[32] S. Salcudean, "A globally convergent angular velocity observer for rigid body motion," IEEE Trans. Auto. Cont., vol. 36, no. 12, pp. 1493-1497, Dec. 1991.

[33] O. Egland and J. Godhaven, "Passivity-based adaptive attitude control of a rigid spacecraft," IEEE Transactions on Automatic Control, vol. 39, pp. 842-846, April 1994.

[34] A. Tayebi and S. McGilvray, "Attitude stabilization of a four-rotor aerial robot: Theory and experiments," To appear in IEEE Transactions on Control Systems Technology, 2006.

[35] R. Mahony, T. Hamel, and J.-M. Pflimlin, "Complimentary filter design on the special orthogonal group $S O(3)$," in Proceedings of the IEEE Conference on Decision and Control, CDC05. Seville, Spain: Institue of Electrical and Electronic Engineers, December 2005.
[36] T. Hamel and R. Mahony, "Attitude estimation on $S O(3)$ based on direct inertial measurements," in International Conference on Robotics and Automation, ICRA2006, 2006.

[37] S. Bonnabel and P. Rouchon, Control and Observer Design for Nonlinear Finite and Infinite Dimensional Systems, ser. Lecture Notes in Control and Information Sciences. Springer-Verlag, 2005, vol. 322, ch. On Invariant Observers, pp. 53-67.

[38] S. Bonnabel, P. Martin, and P. Rouchon, "A non-linear symmetrypreserving observer for velocity-aided inertial navigation," in American Control Conference, Proceedings of the, June 2006, pp. 2910-2914.

[39] R. Murray, Z. Li, and S. Sastry, A mathematical introduction to robotic manipulation. CRC Press, 1994.

[40] H. Khalil, Nonlinear Systems 2nd edition. New Jersey: Prentice Hall, 1996.

[41] L. Lipera, J. Colbourne, M. Tischler, M. Mansur, M. Rotkowitz, and P. Patangui, "The micro craft istar micro-air vehicle: Control system design and testing," in Proc. of the 57th Annual Forum of the American Helicopter Society, Washington DC, USA, May 2001, pp. 1-11.

[42] J. Fleming, T. Jones, P. Gelhausen, and D. Enns, "Improving control system effectiveness for ducted fan vtol uavs operating in crosswinds," in Proc. of the 2nd "Unmanned Unlimited" System. San Diego, USA: AIAA, September 2003.

[43] R. G. Brown and P. Y. C. Hwang, Introduction to Random Signals and Applied Kalman Filtering, 2nd ed. New York, NY: John Wiley and Sons, 1992

[44] J. Thienel, "Nonlinear observer/controller dsigns for spacecraft attitude control systems with uncalibrated gyros," $\mathrm{PhD}$, Faculty of the Graduate School of the University of Maryland, Dep. Aerospace Engineering, 2004.

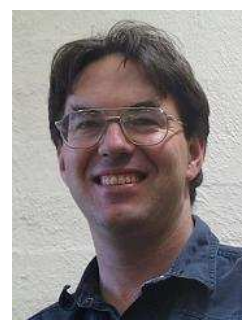

Robert Mahony is currently a reader in the Department of Engineering at the Australian National University. He received a $\mathrm{PhD}$ in 1995 (systems engineering) and a BSc in 1989 (applied mathematics and geology) both from the Australian National University. He worked as a marine seismic geophysicist and an industrial research scientist before completing a two year postdoctoral fellowship in France and a two year Logan Fellowship at Monash University in Australia. He has held his post at ANU since 2001. His research interests are in non-linear control theory with applications in robotics, geometric optimisation techniques and learning theory.

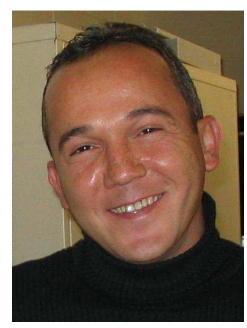

Tarek Hamel received his Bachelor of Engineering from the University of Annaba, Algeria, in 1991. He received his $\mathrm{PhD}$ in Robotics in 1995 from the University of technology Compiègne (UTC), France. After two years as a research assistant at the UTC, he joined the "Centre d'Etudes de Mècanique d'Iles de France" in 1997 as an associate professor. Since 2003, he has been a Professor at the I3S UNSACNRS laboratory of the University of Nice-Sophia Antipolis, France. His research interests include nonlinear control theory, estimation and vision-based control with applications to Unmanned Aerial Vehicles and Mobile Robots.

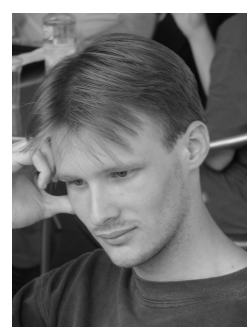

Jean-Michel Pflimlin graduated from Supaero, the French Engineering school in Aeronautics and Space in 2003. He conducted his Ph.D. research at the Laboratory for Analysis and Architecture of Systems, LAAS-CNRS, in Toulouse, France and received the $\mathrm{PhD}$ degree in automatic control from Supaero in 2006. He is currently working at Navigation department of Dassault Aviation in Saint Cloud, Paris. His research interests include nonlinear control and filtering, advanced navigation systems and their applications to Unmanned Aerial Vehicles. 